\title{
Dynactin1 depletion leads to neuromuscular synapse instability and functional abnormalities
}

\author{
Valérie Bercier ${ }^{1,3}$, Jeffrey M. Hubbard ${ }^{2}$, Kevin Fidelin $^{2,4}$, Karine Duroure $^{1}$, Thomas O. Auer $^{1,5}$, Céline Revenu',
} Claire Wyart ${ }^{2^{*}}$ and Filippo Del Bene ${ }^{1 *}$ (I)

\begin{abstract}
Background: Dynactin subunit 1 is the largest subunit of the dynactin complex, an activator of the molecular motor protein complex dynein. Reduced levels of DCTN1 mRNA and protein have been found in sporadic amyotrophic lateral sclerosis (ALS) patients, and mutations have been associated with disease, but the role of this protein in disease pathogenesis is still unknown.

Methods: We characterized a Dynactin1a depletion model in the zebrafish embryo and combined in vivo molecular analysis of primary motor neuron development with live in vivo axonal transport assays in single cells to investigate ALS-related defects. To probe neuromuscular junction (NMJ) function and organization we performed paired motor neuron-muscle electrophysiological recordings and GCaMP calcium imaging in live, intact larvae, and the synapse structure was investigated by electron microscopy.

Results: Here we show that Dynactin1a depletion is sufficient to induce defects in the development of spinal cord motor neurons and in the function of the NMJ. We observe synapse instability, impaired growth of primary motor neurons, and higher failure rates of action potentials at the NMJ. In addition, the embryos display locomotion defects consistent with NMJ dysfunction. Rescue of the observed phenotype by overexpression of wild-type human DCTN1GFP indicates a cell-autonomous mechanism. Synaptic accumulation of DCTN1-GFP, as well as ultrastructural analysis of NMJ synapses exhibiting wider synaptic clefts, support a local role for Dynactin1a in synaptic function. Furthermore, live in vivo analysis of axonal transport and cytoskeleton dynamics in primary motor neurons show that the phenotype reported here is independent of modulation of these processes.
\end{abstract}

Conclusions: Our study reveals a novel role for Dynactin1 in ALS pathogenesis, where it acts cell-autonomously to promote motor neuron synapse stability independently of dynein-mediated axonal transport.

Keywords: Dynactin1, Zebrafish, Neuromuscular junction, Amyotrophic lateral sclerosis, Axonal transport

\section{Background}

ALS is an adult-onset, neurodegenerative disease affecting upper and lower motor neurons. It leads to denervation at the neuromuscular junction, muscle wasting and progressive paralysis, ending in the demise of the patient two to 5 years after diagnosis. Many causative

\footnotetext{
* Correspondence: claire.wyart@icm-institute.org; filippo.del-bene@curie.fr; filippo.del-bene@inserm.fr

${ }^{2}$ Sorbonne Université, Inserm, CNRS, AP-HP, Institut du Cerveau et de la Moelle Épinière, ICM, F-75013 Paris, France

${ }^{1}$ Institut Curie, PSL Research University, INSERM U934, CNRS UMR3215,

Sorbonne Université, F-75005 Paris, France

Full list of author information is available at the end of the article
}

mutations and risk factors have been identified in the last few decades targeting genes like SOD1, TARDBP and FUS, and more recently, C9ORF72, along with multiple pathogenic mechanisms, including protein misfolding and aggregation, impaired RNA metabolism and excitotoxicity (reviewed here [1-5]). Recent evidence revealed multiple cases where an inheritance of risk variants in multiple genes led to disease or altered penetrance of pathogenic mutations. Considering the sporadic nature of most cases, this proposed oligogenic etiology of ALS [4, 6-8] highlights the importance of studies focused on rare variants. Indeed, as ALS remains

(C) The Author(s). 2019 Open Access This article is distributed under the terms of the Creative Commons Attribution 4.0 International License (http://creativecommons.org/licenses/by/4.0/), which permits unrestricted use, distribution, and 
a disease of heterogeneous clinical presentation and variable progression, diagnosed on exclusion, insights into pathophysiological processes could help categorization and lead to the elaboration of effective treatment targeting specific mechanisms.

Axonal transport defects is a common mechanism that has been observed in many neurodegenerative diseases, including ALS [9-11]. Indeed, defects in both fast and slow axonal transport have been reported for cargoes such as mitochondria, endosomes and vesicles containing trophic signaling receptors like TrkB [12-16]. As motor neurons extend long projections to reach their target, they are particularly dependent on axonal transport for communication between the synapse and the cell body, to supply the synapse with building components and to remove cellular detritus for degradation. Axonal transport is mediated by ATP-driven molecular motors, which carry vesicles and organelles by moving along the microtubule network. This component of the cell cytoskeleton is composed of protofilaments polymerized from tubulin subunits which are oriented in a highly polarized manner in the axon, resulting in a fast-growing end $(+)$ at the synapse and a slow-growing end (-) oriented toward the nucleus at the soma. The dynein motor complex transports cargo in the retrograde direction (- end directed) [17] and relies on multi-subunit complexes like dynactin for functional versatility $[18,19]$.

Dynactin subunit 1 (DCTN1, ENSG00000204843; OMIM 601143, hereafter referred to as Dynactin1) is the largest subunit of the dynactin complex and acts as the link between this macromolecular complex and the microtubules via its CAP-Gly domain [20,21]. The dynactin complex regulates the activity of molecular motor complex dynein, where Dynactin1 serves as the link between both complexes [20, 22], and orchestrates the motor's recruitment to the microtubule network at distal ends [20,23]. It has therefore been thought to be essential for dynein function, and for axonal transport [24]. DCTN1 was found to be down regulated in sporadic ALS patients, resulting in a lower protein expression $[25,26]$, suggesting altered expression of this protein could be involved in the pathophysiological process.

Mutations in DCTN1 have also been reported in ALS patients $[8,27-31]$, however their role in motor neuron degeneration is not clear. Indeed, mutations in this gene have previously been found in patients affected with Perry Syndrome, a disease characterized by Parkinsonism. In this case, the reported mutations affect the the $\mathrm{N}$-terminal CAP-Gly domain (G59S) interfere with the incorporation of this subunit within the dynactin complex and leads to the formation of misfolded protein aggregates in a phenotype similar to what is observed following the loss of the whole CAP-Gly domain [32, 33]. In contrast, the mutations reported in ALS cases don't target a particular domain (for instance T1249I, M571 T, R785W, R1101K [28], I196V and R1049Q [30]) and have been found to lead to proper incorporation of Dynactin1 within the dynactin complex without aggregate formation (for M571 T, R785W, R1101K and T1249I [34]). Because of its known function in regulating dynein activity and because axonal transport deficits are a feature of ALS, Dynactin1's implication in neurodegeneration has been assumed to involve axonal transport dysregulation $[5,35]$.

Here, we analyze the effect of Dynactin1 depletion in the zebrafish embryo (genetic inactivation ofortholog dynactin1a ENSDARG00000019743; mutant line mok $^{\mathrm{m} 632}$ ) on the development of the caudal primary $(\mathrm{CaP})$ motor neurons of the spinal cord. Homozygous mutant embryos, referred to as mok ${ }^{\mathrm{m} 632-/-}$ hereafter, initially extend normal CaPs and establish proper neuromuscular junctions (NMJ) with fast-twitch muscle fibers. Depletion of Dynactin1a leads to synapse instability, which impairs further growth and causes electrophysiological dysfunction and locomotor deficits. These defects were found to be independent of changes in axonal transport dynamics or cytoskeletal modulation, two well-known functions relying on Dynactin1. Our results point to a local role for this protein in synapse stability where a protein depletion could contribute to the oligogenic etiology of ALS pathogenesis by inducing NMJ dysfunction without leading to ALS-related motor neuron degeneration in itself.

\section{Material and methods}

\section{Zebrafish husbandry and transgenic lines}

Zebrafish lines were housed in the Curie Institute animal facility, maintained at $28,5^{\circ} \mathrm{C}$ and embryos were raised in egg medium containing methylene blue. All experiments were performed according to the French and European Union animal welfare guidelines, as well as the Curie Institute ethics protocol.

The following transgenic and mutant fish lines were used:

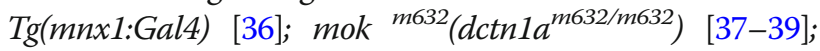
$\operatorname{Tg}($ UAS:GCaMP5G) [40]; $\operatorname{Tg}(c d h 2: C d h 2-G F P)$ [41] .

\section{Molecular cloning \\ 4nrUAS-tagRFPCaax-pA-4nrUAS-eGFP-Rab5c-pA; cmcl2:eGFP; \\ -Rab7-pA;cmcl2:eGFP; \\ -Rab11a-pA;cmcl2:eGFP}

Fusion proteins were generated by fusing $r a b 5 c$, and $r a b 7$ open reading frames from p3'E vectors (kindly provided by Brian Link [42]) with eGFP into pME (Tol2Kit) [43]. UAS constructs were assembled by combining pME-rab-GFP, p3'E-SV4O and a $p 5^{\prime} E$-4nrUAS-tagRFPCaax-pA-4nrUAS vector (containing a membranebound tagRFP reporter under the expression of four 
non-repeated UAS sequences) [44] into a pDestTol2CG \#393 destination vector [43] using the MultiSite Gateway Three-Fragment Vector Construction Kit (ThermoFisher Scientific). The rab11a cDNA was amplified from zebrafish total cDNA using primers $5^{\prime} \mathrm{E}$ - atggggacacgagacgacg and $5^{\prime}$ - ctagatgctctggcagcactg and cloned into a pDONRP2R-P3 to generate a $\mathrm{p} 3$ 'E vector, which was combined with a pME-eGFP vector and a $p 5^{\prime}-4 n r U A S$ tagRFPCaax-pA-4nrUAS vector into a pDestTol2CG \#393 destination vector [43] using the MultiSite Gateway Three-Fragment Vector Construction Kit (ThermoFisher Scientific).

\section{$m n x 1: / y n-G F P-p A$}

A p5'E entry vector was generated by PCR amplification of a $125 \mathrm{bp}$ promoter fragment of the $m n x 1$ gene [36] followed by a BP reaction. The middle entry plasmid was obtained by BP reaction from amplification of two consecutive copies of a sequence encoding the Palmmyr signal of Lyn kinase from Mus musculus (MGCIKSKRKDNLNDDE). The construct was assembled into a pDONR221 using the MultiSite Gateway Three-Fragment Vector Construction Kit (ThermoFisher Scientific) to obtain $m n x 1: l y n-e G F P-p A$.

\section{pUAS-dendra2-rab3-pA}

A middle entry vector carrying dendra2-rab3 was generated by fusing the rab3 open reading frame of $p B H U A S$ Rab3-YFP (kindly provided by Michael Nonet) [45], and the dendra2 sequence from pDendra2-N1 (kindly provided by Jean-René Huynh, Institut Curie, Paris) via PCR amplification. The middle entry vector was combined with a standard $p 5^{\prime} U A S$ vector (Tol2kit) and a standard $p 3$ 'SV4OpA using the MultiSite Gateway Three-Fragment Vector Construction Kit (ThermoFisher Scientific) to obtain pUAS-dendra2-rab3-pApUAS-dendra2-rab3-pA.

\section{pUAS-EB3-meGFP-pA}

The $p M E-E B 3$ plasmid [41] was combined with p3'meGFP under a $U A S$ promoter and assembled into pDONR221 using the MultiSite Gateway ThreeFragment Vector Construction Kit (ThermoFisher Scientific) to obtain $p U A S-E B 3-G F P$.

\section{4xUAS:ubc-EB3-meGFP-E2A-tagRFP-rab3-pA}

This construct was obtained via Gibson assembly using the pT1UciMP Tol1 destination vector previously described. The EB3-meGFP fragment was amplified via PCR from $p U A S-E B 3-m e G F P-p A$, the E2A-tagRFP was amplified via PCR from 4nrUAS-tagRFPCaax-pA4nrUAS-eGFP-Rab5c-pA, and the rab3-pA was amplified via PCR from $p U A S-$ dendra2-rab3-pA. All fragments were inserted after the $u b c$ intron of the $p$ T1UciMP Tol1 destination vector opened by restriction digest with
NcoI-HF (NEB) to obtain 14xUAS:ubc-EB3-meGFPE2A-tagRFP-rab3-pA.

\section{4xUAS:ubc-hDCTN1-eGFP-E2A-tagRFPCaax-pA}

This construct was obtained via Gibson assembly using the pT1UciMP Tol1 destination vector previously described. Dynactin1 was amplified via PCR from a pCDNA3.1 vector containing human Dynactin1-GFP (kindly provided by Stefan Liebau [30]) and fused to $e G F P$ and E2A-tagRFPCaax (described above) using the NEBuilder HiFi DNA Assembly Cloning Kit (NEB). These three sequences were inserted after the $u b c$ intron of the pTIUciMP Tol1 destination vector opened by restriction digest with NcoI-HF (NEB) to obtain 14xUAS: $u b c-h D C T N 1-e G F P-E 2 A-t a g R F P C a a x-p A$.

\section{4xUAS:ubc-ngfra-eGFP-E2A-tagRFPCaax-pA}

Similar to the construct above, the cDNA sequence of the ngfra zebrafish gene (ENSDARG00000088708) coding for the p75 trophic receptor was amplified from zebrafish cDNA via PCR and combined with eGFP and E2A-tagRFPCaax into the pT1UciMP Tol1 destination vector using the NEBuilder HiFi DNA Assembly Cloning Kit (NEB) to obtain 14xUAS:ubc-ngfra-eGFP-E2AtagRFPCaax-pA.

\section{RNA synthesis}

RNA for human Dynactin1-GFP was synthesized from the pCDNA3.1 construct described previously (kindly provided by Stefan Liebau [30]) using the mMESSAGE mMACHINE T7 transcription kit (Invitrogen).

\section{Microinjections}

Embryos were injected at the zygote stage $(1$ cell) using a Picospritzer III pressure ejector and a glass capillary tube pulled with a Flaming-Brown puller as a needle. Injection mixes contained phenol red to judge injected volume and were set to $400 \mathrm{ng} / \mathrm{ul}$ of RNA, and 30-50 ng/ul of recombinant DNA with or without added transposase mRNA (50 ng/ul).

\section{Whole-mount immunohistochemistry}

Embryos were fixed in 4\% paraformaldehyde diluted in PBS for $4 \mathrm{~h}$ at room temperature. They were then rinsed multiple times in PBS containing 0,1\% triton X-100 (PBST) then incubated with a solution of $1 \mathrm{mg} / \mathrm{ml}$ of collagenase (from Clostridium histolyticum, Sigma) in PBS for 20 min (2dpf embryos) or $2 \mathrm{~h}$ (6dpf embryos). The embryos were rinsed several times with PBST then blocked for $1 \mathrm{~h}$ in a block solution containing 1\% bovine serum albumin (BSA), 2\% normal goat serum, $1 \%$ DMSO and $0,1 \%$ triton X-100. The primary antibody was then added with fresh block solution according to the working dilutions listed below, with an incubation 
time of $2 \mathrm{~h}$ at room temperature. After several washes in PBST, the secondary antibody was added in fresh block solution for an incubation of $2 \mathrm{~h}$ at room temperature, then rinsed thoroughly. The embryos were then processed for imaging.

For labelling with conjugated $\alpha$-bungarotoxin, the fixation step was done overnight and the block solution used was composed of $2 \%$ BSA, $0,5 \%$ triton X-100 in PBS. The incubation time for the conjugated $\alpha$ bungarotoxin was $30 \mathrm{~min}$ at room temperature.

\section{List of antibodies}

- Anti-synaptotagmin2 (znp1) (Developmental Studies Hybridoma Bank), monoclonal mouse IgG2a, used at 1:300.

- Conjugated $\alpha$-bungarotoxin-AlexaFluor 594 (ThermoFischer Scientific), $\alpha$-subunit of the nicotinic acetylcholine receptor (AChR) extracted from Bungarus multicinctus venom and conjugated with Alexa Fluor 594 used at $10 \mu \mathrm{g} / \mathrm{ml}$.

- Anti-acetylated tubulin clone 6-11-B-1 (Sigma) purified mouse monoclonal IgG antibody $1,5 \mathrm{mg} / \mathrm{ml}$ used at 1:200.

- Anti-GFP (GeneTex, Euromedex) purified chicken polyclonal IgG antibody, $10 \mathrm{mg} 1 \mathrm{ml}$, used at 1:300.

- Goat anti-chicken Alexa Fluor 488 (Life Technologies) purified goat antibody, used at 1:1000

- Goat anti-mouse Alexa Fluor 488 (Life Technologies) purified goat antibody, used at 1:1000

- Goat anti-mouse Alexa Fluor 635 (Life Technologies) purified goat antibody, used at 1:1000

\section{Touch-evoked escape response assay}

$2 \mathrm{dpf}$ embryos were dechorionated and left to acclimate at room temperature $30 \mathrm{~min}$ prior to the experiment. Each embryo was placed in the center of a $144 \mathrm{~mm}$ petri dish containing egg medium. A refractory period of $30 \mathrm{~s}$ was observed before the presentation of a stimulus. The escape response was elicited by a light brush on the tail of the embryo with a pair of blunt forceps and was recorded with an Olympus FE-5000 camera or with a Sony HDR-AS50 at $30 \mathrm{~Hz}$. The videos were analyzed in ImageJ using the Manual Tracking plugin (Fabrice Cordelières, Institut Curie-Orsay, France).

\section{Morphological images}

Images of the embryos were acquired with a Leica MZ FLIII stereomicroscope (Leica) equipped with a Leica DFC310FX digital camera (Leica).

Fluorescence microscopy for RNA injection validation Images of the GFP signal in the $488 \mathrm{~nm}$ wavelength channel was acquired on a Leica DM 3000 LED microscope equipped with a DMK 33UX250 USB3.0 monochrome industrial camera (The Imaging Source, Bremen, Germany) using Lucia 4.60 software (Laboratory Imaging, Prague, Czech Republic).

\section{Spinning disk confocal microscopy for cell morphology and time-lapse imaging}

We limited our study to $\mathrm{CaP}$ motor neurons within a 4somite window around the cloaca in order to avoid morphological and functional variability that arise between cell types and along the rostro-caudal developmental wave.

Imaging was performed on a Roper confocal spinning disk head mounted on a Zeiss upright microscope, and acquisitions were done with a CoolSNAP HQ2 CDD camera (Photometrics, USA) through the MetaMorph software (Molecular Devices, USA). Embryos were anesthetized using $0.02 \%$ tricaine (MS-222, Sigma) diluted in egg water and embedded in $1 \%$ low melting-point agarose in a glass-bottom cell tissue culture dish (Fluorodish, World Precision Instruments, USA). Acquisitions were done using water immersion long working distance lenses, at 40x magnification (W DIC PL APO VIS-IR; 421,462-9900) for z-stack images of the whole tectum and at 63x magnification (W PL APO VIS-IR (4214809900) for single plane time-lapse imaging of linear axonal segments, and for filopodia imaging. Acquisitions were done using the Metamorph software (Molecular Devices) and resolution in $\mathrm{z}$ was set at $1 \mathrm{um}$ for stacks. Images were assembled and analyzed in ImageJ (NIH). $6 \mathrm{dpf} \mathrm{z}$ - stacks taken in two frames were stitched together using the pairwise stitching function of the Stitching plugin [46].

\section{Time-lapse imaging}

Live imaging of axonal transport was done using fusion proteins combined with a membrane reporter, described previously, expressed in the CaP primary motor neurons by use of the $\operatorname{Tg}(m n x 1: G A L 4)$ line. Time-lapse parameters were determined based on the speed of transport in the spinal cord and set at $1 \mathrm{~s}$ intervals for mitochondria (4nrUAS:tagRFPCaax-pA-4nrUAS:PhbGFP-pA-Tol2; cmcl2:eGFP), for $10 \mathrm{~min}$ total duration, and set at 500 ms for endosomes (rab5c, 7 and 11a), p75, and eb3 comets for $5 \mathrm{~min}$ total duration. For filopodia dynamics time-lapses, $\mathrm{z}$-stacks were taken every $2 \mathrm{~min}$ for $10 \mathrm{~min}$ of total duration.

\section{Kymogram production and analysis}

Time-lapse images were assembled and analyzed in ImageJ. Kymograms were extracted for each time-lapse serie on linear axonal segments using the Kymograph Tool (Montpellier RIO Imaging, CNRS, France), where each 
pixel on the $\mathrm{Y}$ axis represents one timepoint projected against axonal length (X axis).

\section{Calcium imaging during fictive locomotion}

4dpf $\mathrm{Tg}(\mathrm{mnx1}$ :gal4; UAS:GCaMP5G) double transgenic larvae were screened for dense labeling and good expression of GCaMP5 in spinal motor neurons under a dissecting microscope equipped with an epifluorescence lamp (Leica, Wetzlar, Germany). Larvae were anaesthetized in $0.02 \%$ Tricaine-Methiodide (MS-222, Sigma-Aldrich) diluted in fish facility water and mounted on their lateral side in $1.5 \%$ low-melting point agarose in glass-bottom dishes filled with external solution $([\mathrm{NaCl}]=134 \mathrm{mM}$, $[\mathrm{KCl}]=2.9 \mathrm{mM}, \quad[\mathrm{MgCl} 2]=1.2 \mathrm{mM}, \quad[$ HEPES $]=10 \mathrm{mM}$, [glucose $]=10 \mathrm{mM}$ and $[\mathrm{CaCl} 2]=2.1 \mathrm{mM}$; adjusted to $\mathrm{pH}$ 7.7-7.8 with $\mathrm{NaOH}$ and osmolarity 290 mOsm). Larvae were immobilized by injecting $0.1-0.3 \mathrm{~nL}$ of $0.5 \mathrm{mM}$ alpha-Bungarotoxin (Tocris, Bristol, UK) in the ventral axial musculature. Zebrafish larvae were imaged using a custom spinning disk microscope (3i, Intelligent Imaging Innovations, Denver, CO, USA) equipped with a set of water-immersion objectives (Zeiss 20X, 40X, NA =1). Recordings were acquired using Slidebook software at $10 \mathrm{~Hz}$ with a $488 \mathrm{~nm}$ laser. Gain and binning were manually optimized to maximize signal to noise ratio. Z-projection stacks showed full pattern of expression using Fiji (Schindelin et al.,2012). Thin-walled, borosilicate glass capillaries (Sutter Instruments, Novato, CA, USA) were pulled and fire-polished from a Flaming/Brown pipette puller (Sutter Instruments, Novato) to generate water jet stimulation pipettes. Stimulation pipettes were filled with external solution, connected to a pneumatic microinjector with vacuum pressure (WPI, Sarasota, USA), and positioned next to the preparation using motorized micromanipulators under the microscope. Water jet stimulations were either manually induced, or timed online in pClamp8.2 (Axon instruments). Stimulations were elicited every 2-3 min to reduce habituation. Positions of cells along the D$\mathrm{V}$ axis were computed using Fiji and Matlab (Mathworks, USA). Calcium signals were extracted online using custom MATLAB scripts (Kevin Fidelin, Wyart Laboratory, Paris). Regions of interest (ROIs) were manually designed and raw fluorescence signals time series were extracted as the mean fluorescence from individual ROIs at each time point of the recording. DF/F calcium traces were generated and aligned to water jet stimuli in Matlab.

\section{In vivo intracellular recordings}

$6 \mathrm{dpf}$ zebrafish larvae were decapitated and pinned to a Sylgard coated recording chamber (Sylgard 184, Dow Corning, Midland, MI, USA) through the notochord with electrolytically sharpened tungsten pins. The skin was removed and the specimen was bathed briefly in a $10 \%$ formamide solution and subsequently washed in bath recording solution to eliminate spontaneous muscle twitching. For paired recordings, the dura was exposed by suctioning away dorsal muscle fibers with a glass pipette. Typically 3-7 segments of dorsal muscle were removed. Recording electrodes were fashioned from capillary glass (1.5 mm O.D., 1.1 ID, WPI, Sarasota, FL, USA) with a horizontal puller (P1000, Sutter Instruments, Novato, CA). Electrode resistances were 8-14 $\mathrm{M} \Omega$ for CaP motor neurons and 2-5 $\mathrm{M} \Omega$ for fast skeletal muscle fibers. To patch motor neurons, positive pressure $(65 \mathrm{mmHg})$ was applied to the recording electrode via a pneumatic transducer (Fluke Biomedical DPM1B, Everett, WA). Once the electrode was driven through the dura in order to approach the targeted motor neuron, the positive pressure was reduced to 35 $\mathrm{mmHg}$. Fast skeletal muscle fibers were exposed and subsequently patched by first removing the superficial layer of slow muscle fibers with a glass suction pipette. Motor neurons were held at $-65 \mathrm{mV}$ in current clamp mode and 2 msec current injections of $\sim 400 \mathrm{pA}$ were used to evoke action potentials. Muscle cells were held at $-50 \mathrm{mV}$ in voltage clamp mode. External bath recording solution contained the following (in $\mathrm{mM}$ ), $134 \mathrm{NaCl}$, $2.9 \mathrm{KCl}, 2.1 \mathrm{CaCl} 2-\mathrm{H} 20,1.2 \mathrm{MgCl} 2,10$ Glucose, 10 HEPES with $\mathrm{pH}$ adjusted to 7.4 , and osmolarity to 290 mOsm. Motor neuron and muscle cell internal solution contained the following (in $\mathrm{mM}$ ), $115 \mathrm{~K}$-Gluconate, 15 $\mathrm{KCl}, 2 \mathrm{MgCl}$ 2, 0.5 EGTA, 4 Mg-ATP, 10 HEPES pH 7.2, $290 \mathrm{mOsm}$. All reagents were obtained from SigmaAldrich (St. Louis, MO, USA) unless otherwise noted. Patch electrodes contained $40 \mu \mathrm{M}$ Alexa Fluor 488 (Life Technologies Ltd., Paisley, UK). Physiological recordings were made with an Axopatch 700B amplifier and digitized with a Digidata 1440A (Molecular Devices, Fremont, CA, USA). pClamp software (Molecular Devices, Fremont, CA, USA) was used to acquire electrophysiological data. Motor neuron recordings were acquired at a sampling rate of $50 \mathrm{kHz}$ and postsynaptic currents were acquired at $100 \mathrm{kHz}$. Recordings were low pass filtered at $2.2 \mathrm{kHz}$. Series resistance was monitored for muscle cell recordings and was $<10 \mathrm{M} \Omega$. Data were analyzed with Clampfit (Molecular Devices, Fremont, CA, USA), Igor Pro 6.34 (WaveMetrics, Lake Oswego, OR), and Excel 2010 (Microsoft, Redmond, WA, USA). Summary data are presented as average \pm SEM.

\section{Electron microscopy}

$6 \mathrm{dpf}$ larvae were fixed in $2 \%$ glutaraldehyde and $2 \%$ paraformaldehyde in cacodylate buffer $0.1 \mathrm{M}$ pH 7.4 to which $3 \mathrm{mM}$ of $\mathrm{CaCl} 2$ was added for $2 \mathrm{~h}$ at RT. Samples were washed 3 times in cacodylate buffer $0.1 \mathrm{M}$ pH 7.4 and then post-fixed with $1 \%$ osmium tetroxide in distilled water for $1 \mathrm{~h}$ at $4{ }^{\circ} \mathrm{C}$. After an extensive wash $(3 \times$ $10 \mathrm{~min}$ ) with distilled water they were incubated for $1 \mathrm{~h}$ 
in $5 \%$ uranyl acetate in water. They were then dehydrated in a graded series of ethanol solutions $(2 \times 5 \mathrm{~min}$ each): 50, 70, 80, 90, and 100\%. Final dehydration was performed twice in $100 \%$ acetone for $20 \mathrm{~min}$. Samples were then progressively infiltrated with an epoxy resin, Epon $812^{\circ}$ (EMS, Souffelweyersheim, France): 1 night in $50 \%$ resin $50 \%$ acetone at $4{ }^{\circ} \mathrm{C}$ in an airtight container, $2 \mathrm{x} 2 \mathrm{~h}$ in pure fresh resin at room temperature. They were embedded in the bottom of capsules (Beems size 3, Oxford Instruments, Saclay, France) and the resin was polymerized at $60^{\circ} \mathrm{C}$ for $48 \mathrm{~h}$ in a dry oven. Blocks were cut with an UC7 ultramicrotome (Leica, Leica Microsystemes SAS, Nanterre, France). Semi-thin sections ( $0.5 \mu \mathrm{m}$ thick) were stained with $1 \%$ toluidine blue in $1 \%$ borax. Ultra-thin sections $(70 \mathrm{~nm}$ thick) were recovered either on copper (conventional morphology) or nickel (immunoelectron microscopy) grids and contrasted Reynold's lead citrate. Ultrathin sections were observed with a Hitachi HT7700 electron microscope (Elexience, Verrière-le-Buisson, France) operating at $70 \mathrm{kV}$. Images were taken with an AMT41B camera at low $(\times 53,000)$, medium $(\times 70,000)$, and high $(\times 110,000)$ magnification, the last of which was used for quantification, done with ImageJ.

\section{Quantitative RT-PCR}

Total RNA was extracted from previously phenotyped 6dpf embryos using a standard TRIzol reagent protocol (ThermoFisher Scientific). cDNA was then synthesized using the retrotranscription SuperScript III First-Strand Synthesis system kit (ThermoFisher Scientific) with the random hexamer primers. The qRT-PCR mix was prepared in technical triplicates with SYBR Green Master Mix (ThermoFisher Scientific) and run on an ABI PRISM 7900HT Real-Time PCR System (ThermoFisher Scientific) using ef1 $a$ and $r p l 13 a$ as reference genes [47]. The analysis was performed according to the deltaCT quantification method and presented as a relation to wild-type levels (fold-change) [48].

\section{List of q RT-PCR primers}

dctn1a_Fwd: TCGAAGCTGA TGATCCCGTG dctn1a_Rev: TCCTGAGGGA GTGTGTGTGA dctn1b-fwd: GCAAAGGAGG AGAAGAGAGG dctn1b-rev: TGGAGAAGGC GATGGAC P22P24_Fwd: CACAAATACA CATTCAACAG CAGGAC

P22P24_Rev: AGAGTTTCAT CCCACTGTGA AAAC TG

P25_Fwd: CTGTCCTTCC CCCAGAGACA P25_Rev: TCTGGCTGAG AGGGAGGAAT p50_Fwd: CCTCCAACGA GCCTGATGTT p50_Rev: TAGCGCTGAC GTGTTTGTCT
ndel1b_Fwd: TACACCTGTG GGGAAGACCA ndel1b_Rev: TCCTTGCTGC CTGATCCTTG pafah1b1a_Fwd: CTTGTGCACC CTGGAGGAAA pafah1b1a_Rev: GTACGGAGCA GTCTTGTGGA pafah1b1b_Fwd: TGACACTGGT TGGCCATGAT pafah1b1b_Rev: AGTGTTCATG GGCACTGAGG bdnf_Fwd: CTTGAGGTGG AAGGGGAAGC G bdnf_Rev: GTAACGGCGG CTCCAAAGGC actr1.1-1_Fwd: GGGTCGGGAG TTATCAAGGC actr1.1-1_Rev: CCGGTGCTCCTCTGCTTTAG kif14_Fwd: CTCCAGCACA CCTCATGGAG kif14_Rev: TCCCTGGAGC TGAAAGGTCT rpl13a_Fwd: TCTGGAGGACTG TAAGAGGTTGC rpl13a_Rev: AGACGCACAATC TTGAGAGCAG ef1a_Fwd: CTGGAGGCCAGC TCAAACAT ef1a_Rev: ATCAAGAAGAGT AGTA CCGCTAGCATTAC

\section{Statistics}

Data compilation and analysis were done using Excel (Microsoft, USA) and graph generation was done using GraphPad Prism version 6 for Windows (GraphPad Software, USA). Using GraphPad, Student's t-test was used for normally distributed data and Mann-Whitney U test was used for non-normally distributed data, when comparing mutants and wild-types. For comparison of multiple groups, SigmaPlot 11.0 integrated with SigmaStat 3.1 was used and a one-way ANOVA on ranks was performed, followed by Dunn's multiple comparison procedure (pairwise). The Z-test was used to compare population proportions. Significance, set at $p \leq 0,05(*)$, $p \leq 0,01\left(^{(* *)}, p \leq 0,001{ }^{(* * *)}\right.$.

\section{Results}

Establishing a model for Dynactin1 depletion

Previous work has yielded mikre oko ${ }^{m 632}\left(m^{2} k^{m 632}\right)$ a transgenic line harboring a point mutation within the coding sequence for dynactin1a, a zebrafish ortholog for DCTN1 [37]. The $\mathrm{C}$ to $\mathrm{T}$ transition at nucleotide 2395 creates a premature stop codon and severely reduced dynactin1a mRNA in $m o k^{m 632-/-}$ embryos [39]. A subsequent absence of a detectable truncated peptide suggests the mutation is amorphic or strongly hypomorphic [49]. Homozygous mutants larvae do not survive past the second week of development, as has been described before [38]. As Dynactin1 loss-of-function has proven to be embryonic lethal in other models $[50,51]$, this suggests that maternal contribution of Dynactin1a in the zebrafish model is sufficient to ensure early survival. Maternally-provided protein is depleted by $4 \mathrm{dpf}$ [52] and we observed a nearly $70 \%$ reduction in protein level in homozygous embryos at $2 \mathrm{dpf}$ (Additional file 1: Figure S1b, c). This level is comparable to what has been 
achieved by cell culture siRNA silencing of DCTN1 in previous studies [53].

At early stages, $m o k^{m 632-/-}$ embryos are indistinguishable from their wild-type siblings, and by 4 days postfertilization (dpf) exhibit smaller eyes with protruding lenses, a morphological phenotype resulting from cell death in the photoreceptor layer, also rendering them blind [38]. No additional morphological phenotype was visible in 6dpf homozygous mutant larvae (Additional file 1: Figure S1a) and heterozygous carriers are adult-viable and morphologically indistinguishable from wild-type siblings.

Dynactin1a depletion does not affect initial development of the CaP motor neurons, but leads to growth defects and abnormal innervation of fast-twitch muscle fibers

In order to determine if reduced levels of Dynactin1a had an effect on the development of motor neurons, led to their degeneration, or impaired their function, we focused our attention on the trunk and tail, more specifically, the CaP motor neurons of the spinal cord [54]. The CaPs innervate fatigable type II (fast-twitch) muscle fibers as early as $22-24 \mathrm{~h}$ post-fertilization (hpf), forming an early but functional NMJ by $2 \mathrm{dpf}$ [55].

We visualized $\mathrm{CaP}$ morphology in live embryos by single-cell expression of a membrane-bound fluorophore. This was achieved by injection in zygotes of a DNA construct where lyn-GFP was put under the control of the $m n x 1$ transcription factor, selectively expressed in postmitotic motor neurons of the spinal cord [56]. Comparing cell tracings in $\mathrm{mok}^{\mathrm{m632-/-}} \mathrm{em-}$ bryos with their wild-type siblings revealed that the complexity of axonal arbors, defined by total cell length, projection number, and complexity by branch order, was not altered at $2 \mathrm{dpf}$ (Fig. 1a, b). CaPs in $6 \mathrm{dpf} \mathrm{mok}^{\mathrm{m} 632-/-}$ larvae exhibited a significantly smaller arbor, which was also less complex, as determined by a reduced total cell length and number of projections (Fig. 1c, d).

We next examined NMJ integrity by performing doubleimmunohistochemistry on fixed embryos. Presynaptic structure was revealed by labeling of Synaptotagmin-2 (Additional file 2: Figure S2a, c, in green) and the postsynaptic receptors were labeled by a fluorophore-conjugated $\alpha$-bungarotoxin (Additional file 2: Figure S2a, c, in red), which binds irreversibly to the acetylcholine receptors (AChR) present on muscle fibers. Analysis revealed no change in colocalization or correlation of both signals in the 2dpf ventral root (Additional file 2: Figure S2b), indicating that pre- and postsynaptic components are well aligned and that $\mathrm{CaPs}$, along with the other motor neurons present in the ventral root, properly innervated their target muscle in $m o k^{m 632-/-}$ embryos at 2dpf. These results indicate that Dynactin1a depletion does not interfere with initial development of the CaPs, as the cell size and synaptic structure of the NMJ are conserved in $2 \mathrm{dpf} m o k^{m 632-/-}$ embryos.
However, we found reduced coverage of pre- and postsynaptic markers in 6dpf $\mathrm{mok}^{\mathrm{m} 632-/-}$ larvae, consistent with the observed smaller arbor, but also reduced colocalization of both markers as shown by lower Pearson's and overlap coefficients (Additional file 2: Figure S2d). As we did not detect orphan vesicles or receptors at $6 \mathrm{dpf}$, our results suggest that the smaller arbor size is due to improper growth rather than degeneration.

These results indicate that depletion of Dynactin1a leads to proper migration and initial development of $\mathrm{CaP}$ motor neurons and their NMJ, but to growth defects at $6 \mathrm{dpf}$ associated with a compromised NMJ structural integrity.

\section{Dynactin1a depletion does not alter distribution of cargoes or axonal transport dynamics}

Since Dynactin1 is thought to regulate and direct dynein activity, and acts as the dynactin complex's only direct link with microtubules, we hypothesized that if dyneinmediated retrograde transport was reliant on Dynactin1a as an essential part of the dynactin complex, a depletion could reduce the number of assembled dynactin complexes available and cause defects in clearing of damaged organelles and detritus, or could affect signaling from the synapse. Alternatively, as Dynactin1 is known to be involved in the coordination of bidirectional movement [57], depletion could affect supply for a growing synapse by anterograde transport. We then sought to determine if Dynactin1a depletion caused axonal transport defects in $\mathrm{CaP}$ motor neurons of $2 \mathrm{dpf}$ embryos, and if this could be the cause of the reduced arbor size observed at $6 \mathrm{dpf}$. To exclude the influence of an aberrant morphology on this process, we focused this analysis on $2 \mathrm{dpf}$ embryos, which still present normal CaP morphology,

We selected general cargo markers and generated fusion protein constructs to analyze the axonal transport dynamics of mitochondria (labeled by phb-GFP) [44], early endosomes (labeled by rab5c-GFP), late endosomes/multivesicular bodies (labeled by rab7-GFP), and recycling endosomes (labeled by rab11a-GFP) in vivo. To effectively target $\mathrm{CaP}$ motor neurons, we used the GAL4/UAS system and relied on injection of DNA constructs in the $\operatorname{Tg}(m n x 1: G A L 4)$ transgenic background with a co-expressed membrane-bound fluorophore reporter (UAS:tagRFP-Caax) to confirm cell type by morphology (Additional file 3: Figure S3a).

We first assessed cargo distribution in CaP cells, as disruption of retrograde transport could lead to the formation of aggregates or to abnormal distribution, where cargo would amass at one end of the distal end of the cell if only being transported anterogradely. Furthermore, transport defects could affect the fusion and fission of endosomal vesicles and mitochondria, a process that is essential to their function and if impaired, would 

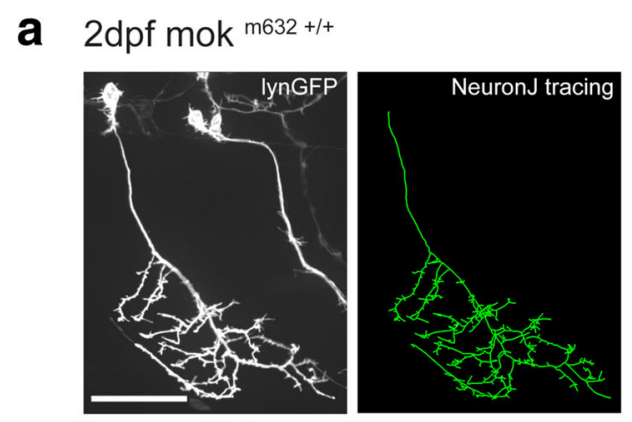

2dpf mok m632--

b
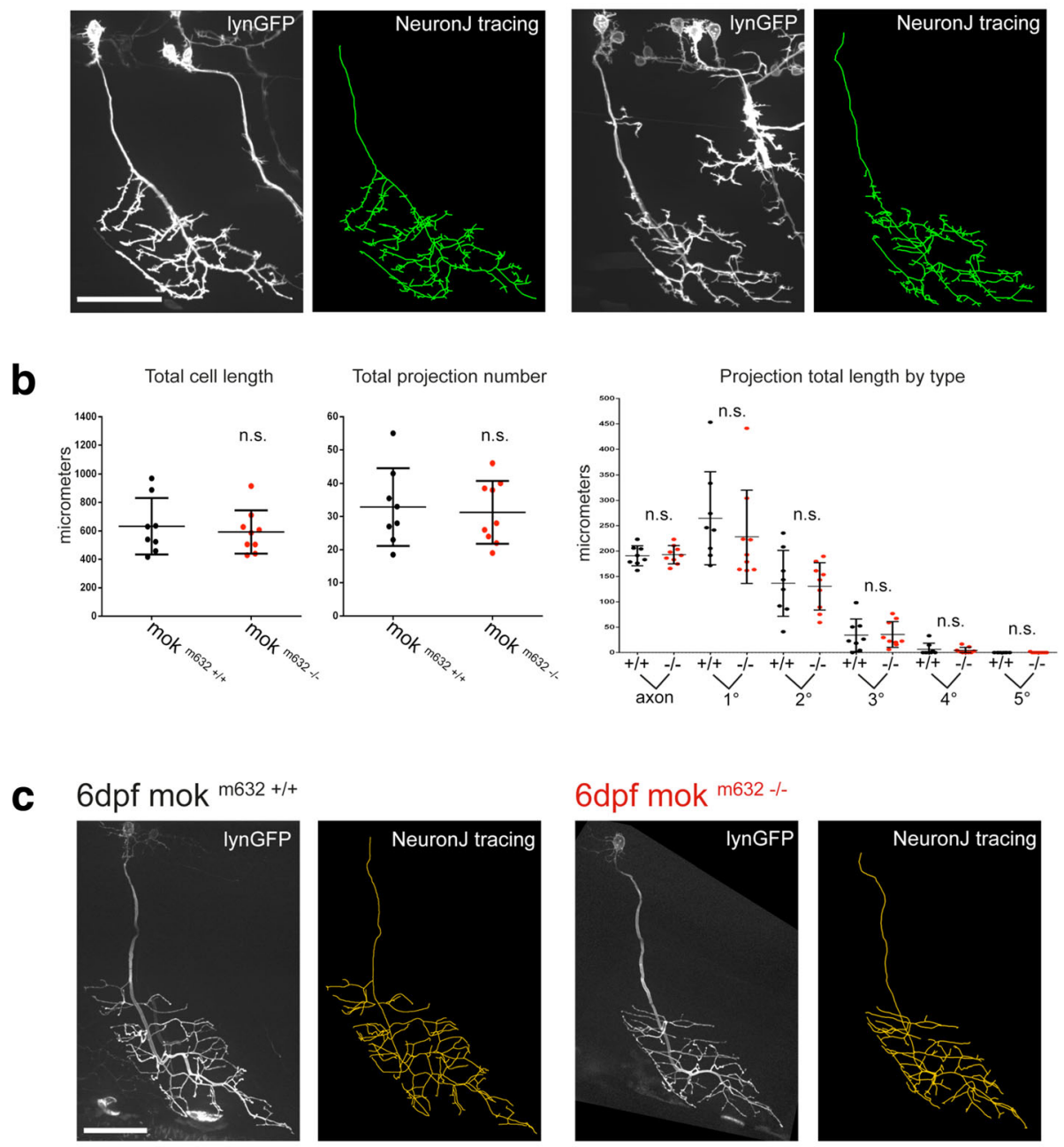

6 dpf mok m632--
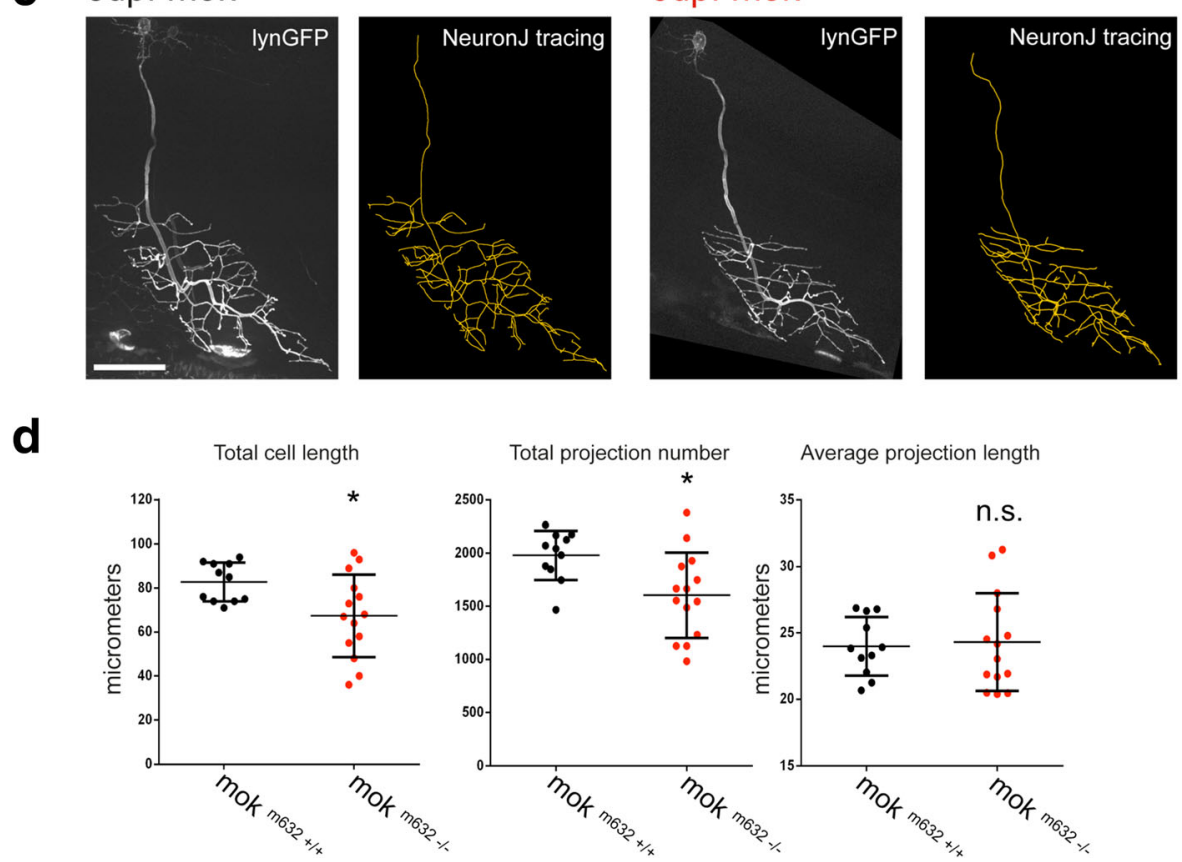

Fig. 1 Dynactin1a depletion leads to proper initial development of the CaPs but impaired growth. Axonal morphology of CaP primary motor neurons in vivo at a 2dpf, and at $\mathbf{b} 6 \mathrm{dpf}$ visualized in confocal z-stack projections by single-cell expression of membrane-bound mnx1:lyn-GFP, with NeuronJ tracings of the axonal arbor. Quantification of the tracings for size (total cell length and projection number) and complexity (projection number by branch order) of CaPs, c showing no significant difference in size between cells of mutants and wild-type siblings at 2dpf, but $\mathbf{d}$ revealing that CaPs in 6dpf homozygous mutant larvae have a smaller arbor composed of fewer projections, which retain average length when compared with their wildtype siblings. All data presented as average $+/-$ SD. (b: $n$ cells wild-type, mutant $=9$, 9; $\mathbf{d}: \mathbf{n}$ cells $=11,14$ ). Scale bar $=50 \mu \mathrm{m}$

alter their size. We quantified number, mean area, total area (coverage) of all labeled cargoes, as well as their axonal distribution with relation to the cell body in $2 \mathrm{dpf}$
CaPs (Additional file 3: Figure S3b). No significant difference was observed for these metrics in mok ${ }^{\mathrm{m} 632-/-}$ embryos when compared with their wild-type siblings, 
and no aggregates were seen, indicating a normal distribution of cargoes.

In order to determine if Dynactin1a depletion modified axonal transport dynamics, we performed in vivo time-lapse imaging of single $\mathrm{CaP}$ axonal segments, located at mid-axon. This allowed the quantification of axonal transport of cargoes in single cells, by kymogram analysis of transport (Additional file 4: Figure S4a). We first classified each cargo trace into three net transport states based on their movement during the acquisition period, whether they were immobile (black), moving toward the cell body (retrograde, magenta) or moving toward the synapse (anterograde, cyan). We found no significant difference in the percentage of cargo in each state between mok ${ }^{\mathrm{m} 632-/-}$ embryos and their wild-type siblings (Additional file 4: Figure S4b). We then quantified metrics such as area flux in the retrograde direction and in the anterograde direction (Additional file 4: Figure S4c, d), and axonal segment vesicle density (Additional file 4: Figure S4e). In addition, we also determined average run speed, length, and duration for runs in both the retrograde and the anterograde direction (example traces on Additional file 4: Figure S4a; Additional file 5: Figure S5). Surprisingly, we did not find any significant difference in these measurements when comparing mok ${ }^{\mathrm{m} 632-/-}$ embryos with their wildtype siblings.

These results suggest that the extent of Dynactin1a depletion at $2 \mathrm{dpf}$ does not affect distribution, anterograde or retrograde axonal transport of mitochondria, and early, late, and recycling endosomes in $\mathrm{CaP}$ motor neurons.

\section{Cytoskeleton dynamics are not affected by Dynactin1a depletion}

The growth defects observed at $6 \mathrm{dpf}$ could be due to the switch in growth signals between a migrating axon, directed by guidance cues [58, 59], and a maturing arbor, responding to local trophic signaling [60, 61]. Indeed, trophic signaling is essential for the growth and plasticity of $\mathrm{CaP}$ arbors. The actin cytoskeleton will form filopodia to search the environment for guidance cues, trophic signaling and adhesion molecules provided by the muscle fibers [62]. These structures get infiltrated by microtubules to form nascent branches, once stabilized by post-synaptic partners, [63], or are retracted when proper signal is absent [64]. Proteins localizing at microtubule +ends (like Dynactin1) are known to regulate the actin cytoskeleton and mediate the signaling of guidance cues during neuronal development [65].

In order to determine if Dynactin1a depletion interfered with the actin cytoskeleton stabilization or with trophic signaling, we monitored filopodia dynamics via in vivo time-lapse imaging of $\mathrm{CaP}$ cells expressing
lynGFP (Fig. 2a). We quantified the amount of unstable filopodial extensions by means of total length, total number and average length of unstable filopodia at 1dpf, $2 \mathrm{dpf}, 3 \mathrm{dpf}$ and $4 \mathrm{dpf}$, but found no significant differences between mok m632-/- embryos and their wild-type siblings aside from a slight reduction in total length of unstable filopodia at $2 \mathrm{dpf}$ (Fig. 2b). To confirm that loss of Dynactin1a did not alter trophic signaling, we also performed axonal transport analysis of vesicles containing the survival/suicide low-affinity trophic receptor p75 (ngfra), and found no difference in ratios, area flux or transport metrics (Additional file 6: Figure S6). Because we only observed a slight reduction in total length of unstable filopodia at $2 \mathrm{dpf}$, without additional defects in other metrics like average length and filopodia number, as well as no in changes trophic signaling, we deemed this change not biologically relevant and moved on to the analysis of the microtubule cytoskeleton.

As Dynactin1 is known to bind microtubules via its CAP-Gly domain, and to act at as an anti-catastrophe factor at plus-tips (+tips) [66], we then investigated microtubule growth by quantification of EB3 comets. This +tip protein binds the labile end of the tubules during bouts of assembly and the resulting runs, or "comets" were visualized in vivo by time-lapse imaging of fusion proteins in single $\mathrm{CaP}$ arbors at $2 \mathrm{dpf}$ and $6 \mathrm{dpf}$ (Fig. 2c). Quantification of comet metrics like average distance, time and speed of runs, did not reveal any changes in either timepoints when comparing mok ${ }^{\mathrm{m} 632}$ ${ }^{-/-}$embryos with their wild-type siblings (Fig. 2d), suggesting microtubule growth and stability are not affected by Dynactin1a depletion.

Microtubule capture at synapses is known to rely on the dynein/dynactin complex and its interaction with adhesion molecules to anchor microtubules at the membrane and promote synapse stability [67]. We therefore co-expressed our EB3 comet construct described previously with rab3-tagRFP, a marker for putative synapses [45], to label both growing microtubules and putative synapses within the same $\mathrm{CaP}$ arbor in live $2 \mathrm{dpf}$ and $6 \mathrm{dpf}$ embryos (Fig. 2e). We then performed time-lapse imaging to determine the density of terminating comets and of synapses in terminal branches of the axonal arbor. The ratio of microtubule capture, defined by comets terminating their run at putative synapse sites, for mok m632-/- embryos was unchanged when compared with their wild-type siblings (Fig. 2f). This suggests that synaptic microtubule capture, while dependent on interaction between dynein and the dynactin complex [67], is not affected by Dynactin1a depletion.

These results indicate that the growth defects observed in 6dpf $\mathrm{CaP}$ motor neurons upon Dynactin1a depletion do not result from impaired modulation of the actin or 

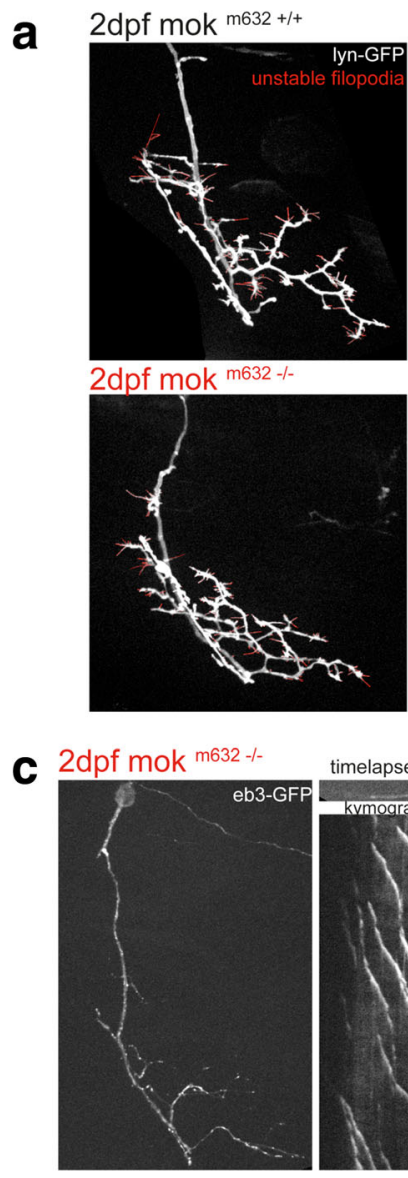

timelapse $\mathrm{t}=0$

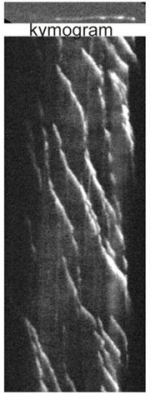

d
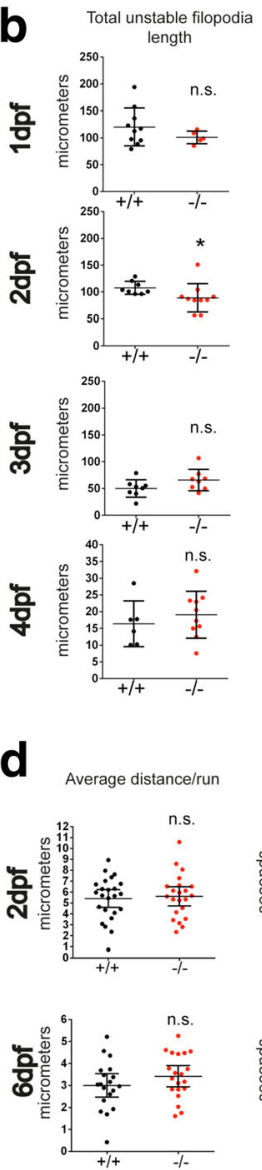

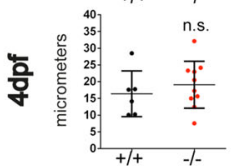

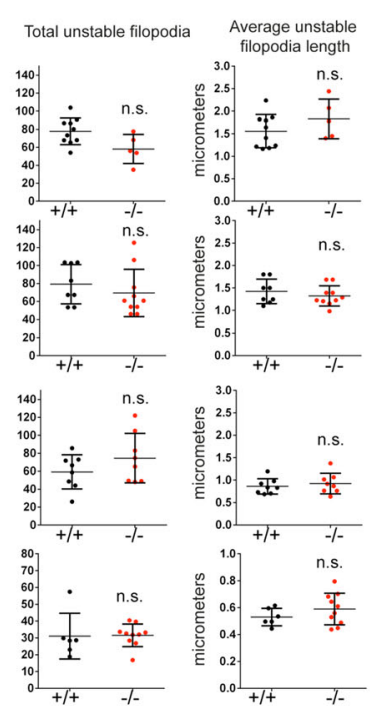

e 2


f
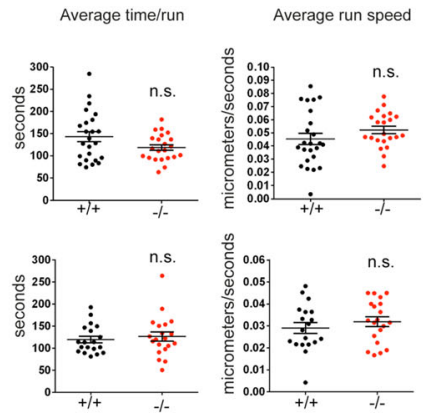
Percentage of comets
terminating at synapses
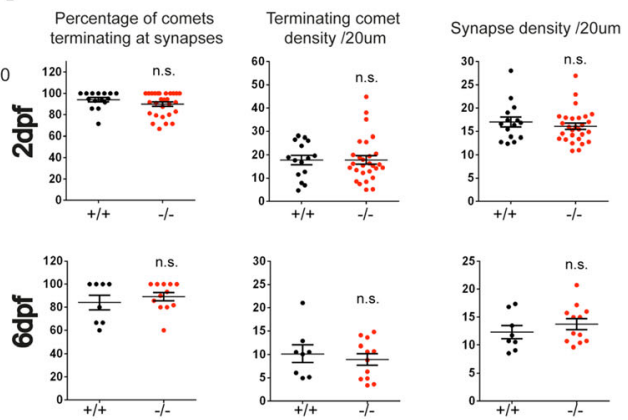

Fig. 2 CaP growth defect is independent of cytoskeleton dynamics modulation. a Actin filopodia dynamics is assayed by time-lapse imaging of single CaP cell arbors expressing membrane-bound reporter lyn-GFP, from 2dpf to 4dpf. Example of confocal z-stack projection of a $2 \mathrm{dpf} \mathrm{CaP}$, with an overlay showing total unstable filopodia in red. b Quantification of filopodia dynamics over 10 min reveals no change in total unstable filopodia length, number or average length for $1 \mathrm{dpf}, 2 \mathrm{dpf}$, $3 \mathrm{dpf}$ or $4 \mathrm{dpf}$ cells, with the exception of total unstable filopodia length at $2 \mathrm{dpf}$, which was found to be slightly diminished. c Microtubule growth was determined by time-lapse imaging of eb3-GFP comets at both 2dpf and 6dpf. $\mathbf{d}$ Quantification of extracted kymograms shows no change in microtubule growth at either timepoint, as determined by average distance, duration and average speed of comet runs. e Microtubule capture at putative synapses was assayed by expression of a synaptic marker (rab3-tagRFP, in red) simultaneously with eb3-GFP (in green) at $2 \mathrm{dpf}$ and $6 \mathrm{dpf}$. $\mathbf{f}$ Quantification of microtubule capture at putative synapses, density of terminating eb3 comets or putative synapses per axonal segment reveal this process was not affected by loss of Dynactin1a. Data presented as average $+/-$ SEM. (b: $1 \mathrm{dpf} n=10,5 ; 2 \mathrm{dpf} n=8,10,3 \mathrm{dpf} n=8,8,4 \mathrm{dpf} n=6,10$; d: $2 \mathrm{dpf} n=24,22,6 \mathrm{dpf} n=22,20 ; \mathbf{f}: 2 \mathrm{dpf} n=15,28,6 \mathrm{dpf} n=8,12$ ) 
microtubule cytoskeleton, and that the cell has both the potential for sensing and the support for trophic signaling in mok ${ }^{\mathrm{m} 632-/-}$ embryos.

\section{Dynactin1a depletion leads to synapse instability at the NMJ}

Synapses are known to be necessary for the stabilization of new branches in a growing axonal arbor [67] and previous studies suggested a role for Dynactin1a in synapse growth and stability in Drosophila [68, 69]. While we did not observe a change in microtubule capture, defects in local organization of the presynaptic structure could lead to instability and impair growth of the CaPs.

Double immunohistochemistry on whole-mount preparations revealed overall conserved NMJ structural integrity for all motor neurons present in the ventral root at 2dpf (Additional file 2: Figure S2 a, b). In order to observe $\mathrm{CaP}$ synapses specifically, we labeled single neurons by injecting pUAS-dendra2-rab3 in the $\operatorname{Tg}(m n x 1$ : GAL4) transgenic background. We observed the size and coverage of putative synapses in single cells of live mok m632-/- and wild-type embryos at 2dpf (Additional file 7: Figure S7a), where no difference was found in number, average size, and arbor coverage (total area) of putative synapses between mok m632-/- embryos and their wildtype siblings (Additional file 7: Figure S7b). However, labeling of CaP synapses in live 6dpf larvae (Fig. 3a) revealed that the smaller cell arbors seen in mok m632-Iembryos contain less synapses, which are also of smaller size (Fig. 3b).

We then performed time-lapse imaging on $\mathrm{CaP}$ arbors over a period of $3 \mathrm{~h}$, a benchmark lifetime for putative synapse stabilization [67] (Fig. 3c) in order to assess if Dynactin1a depletion led to instability at $2 \mathrm{dpf}$ which could explain the reduced number of NMJ synapses in $6 \mathrm{dpf}$ arbors. While we had not found significant differences for number of synapses between wild-type and mok m632-/- embryos at 2dpf (as shown in Additional file 7: Figure S7b), comparison of the same cell between the two timepoints (initially and $3 \mathrm{~h}$ later) allowed quantification of synapses lost and gained over this period, represented as fold-change. We found reduction in number and arbor coverage (total area) of putative synapses in $2 \mathrm{dpf}$ mok m632-/- embryos when compared with their wild-type siblings, while average size (mean area) was maintained (Fig. 3d). In addition to microtubule capture, which was found to be unchanged in our mutant (Fig. 2e, f), synapse stability also relies on interaction with adhesion molecules. We investigated localization of N-Cadherin at NMJ synapses by using previously described BAC transgenic $T g(c d h 2: C d h 2-G F P)$ [41]. At the NMJ, N-Cad-GFP forms a puncta at the center of the presynaptic structure and this localization was not perturbed in $2 \mathrm{dpf}$ (Additional file 8: Figure S8a) or 6dpf mok ${ }^{\text {m632-I- }}$ embryos (Additional file 8: Figure $\mathrm{S} 8 \mathrm{~b})$. As N-Cadherin is known to be involved in synapse stabilization by mediating cell-cell interactions, our results suggest that synapse instability is not due to impaired localization of this adhesion molecule at the synapse, although defects could still arise from compromised interactions upon depletion of Dynactin1a.

These results indicate that Dynactin1a depletion impairs synapse stability at $2 \mathrm{dpf}$, independently of proper localization of $\mathrm{N}$-Cadherin, and leads to impaired stabilization of nascent branches of growing $\mathrm{CaP}$ motor neuron arbors and a reduced number of putative synapses at $6 \mathrm{dpf}$.

\section{Ultrastructural analysis of NMJ synapses support a local role for Dynactin1a}

Visualization of the 6dpf NMJ by electron microscopy (Fig. 3e) confirmed the lack of aggregates in synaptic terminals of mok ${ }^{\mathrm{m} 632-/-}$ larvae which could have supported defects in axonal transport. In addition, we found that the synaptic vesicles were of similar average size and density throughout the terminal in both mutant and wild-type larvae (Fig. 3f). Although they appeared less clustered, it was not possible to determine if organization of vesicle pools was maintained in mok m632-I- larvae. When looking at the active zone (AZ) perimeter (yellow circle Fig. 3e), we observed normal density of synaptic vesicles, which exhibited a similar distribution in relation to the center of the AZ (Fig. 3g), suggesting no change in the availability of vesicles as part of the readily-releasable pool. However, the synaptic cleft as measured between the neuron membrane and the muscle at the level of the AZs, was significantly wider in mok ${ }^{\mathrm{m} 632-/-}$ larvae (Fig. 3g). These observations indicate that despite the lack of changes in microtubule capture at synapses or in the localization of N-cadherin, Dynactin1a could have a role in active zone structure and organization.

\section{Synapse instability leads to impaired NMJ function and locomotion defects}

To determine if synapse instability and wider synaptic clefts had functional consequences on synaptic transmission, we performed whole-cell voltage clamp recordings of individual fast-twitch skeletal muscle fibers and monitored spontaneous miniature end plate currents (mEPCs, Fig. 4a). We observed that mEPCs from the muscle of 6dpf mok ${ }^{\mathrm{m} 632-/-}$ larvae and of their wild-type siblings shared similar kinetics (Fig. 4b, Additional file 9: Figure S9a, b), frequencies (Fig. 4c) and amplitudes (Fig. 4d) of $\mathrm{mEPC}$, as well as a similar quantal size for spontaneously released single vesicles from the $\mathrm{CaP}$ [70] (Fig. 4e), suggesting that even with severely reduced levels of Dynactin1a, the synaptic vesicles in CaPs motor neurons 


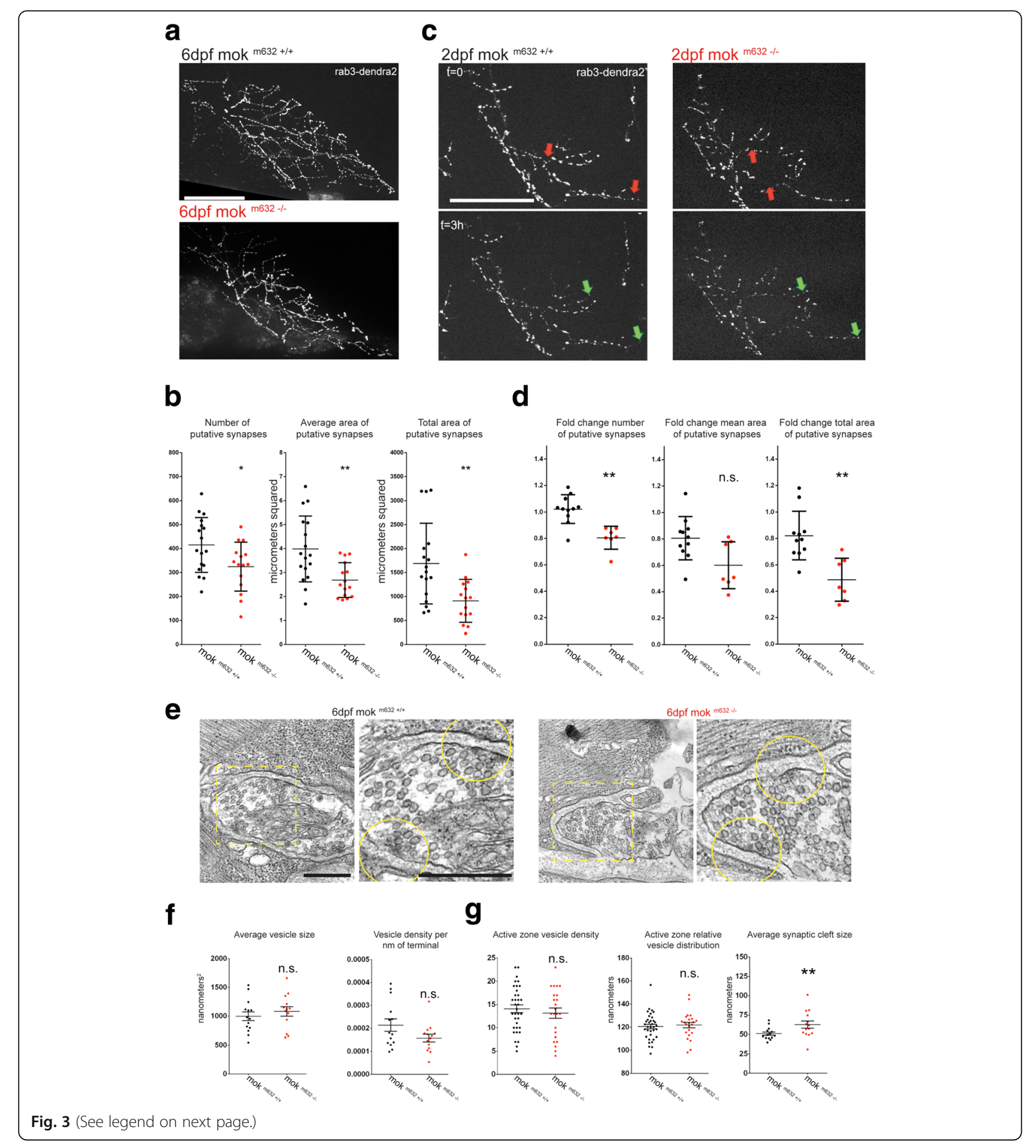




\section{(See figure on previous page.)}

Fig. 3 Dynactin1a depletion leads to synapse instability at $2 \mathrm{dpf}$, reduced synaptic density at $6 \mathrm{dpf}$ and ultrastructural changes. a Putative synapses are visualized with rab3-dendra2 labeling in single CaP cells at $6 \mathrm{dpf}$. b Synaptic coverage in the arbors, determined by number, average area and total area, of putative synapses is reduced in 6dpf larvae in homozygous mutant embryos when compared with their wild-type siblings. c Synapse stability at $2 \mathrm{dpf}$ was assayed by imaging cell arbors over a period of $3 \mathrm{~h}$, where comparison of the initial stack $(t=0)$ with the subsequent one $(t=3 \mathrm{~h})$ for the same cell was used to determine the number of stable synapses. Examples of synapses added and lost are indicated in green and red arrows respectively. d Quantification is presented as fold-change and reduced in homozygous mutant embryos for number and total area, but not for mean area of putative synapses, when compared with their wild-type siblings. e Electron micrograph of a transverse section of the 6dpf NMJ, with close-up (dashed yellow box), showing active zones (center of yellow circle) in NMJ synapses of mok ${ }^{\text {m632--- }}$ larvae and their wild-type siblings. $\mathbf{f}$ No changes were detected in number of synaptic vesicles and average vesicle size when measured in the synaptic terminal. $\mathbf{g}$ Normal density and distribution of vesicles was also observed around the active zones (yellow circle perimeter), however the synaptic clefts were significantly wider at mok ${ }^{\text {m632- }}{ }^{-}$lanvae active zones. Data shown as $\mathbf{b}$ d) average +/-SD, f) g) average +/- SEM. (c: $n$ cells $=11,7 ; \mathbf{d}$ : $n$ cells $=17,15$, f: $n$ slices $=14,14 ; \mathbf{g}: \mathrm{n}$ active zones $=34,22)$. Scale bar a) c) $50 \mu \mathrm{m}$; e) $500 \mathrm{~nm}$

are released normally, that they contain usual quantities of neurotransmitter and that the postsynaptic ACh receptors on fast-twitch muscle fibers are not affected.

In order to investigate the consequence of Dynactin1a depletion on evoked neurotransmission, we next performed paired whole-cell patch clamp recordings of $\mathrm{CaP}$ motor neurons and of their target fast-twitch skeletal muscle [70] (Fig. 4f). In current clamp mode, a short pulse of current ( $2 \mathrm{msec}, \sim 400 \mathrm{pA})$ was injected into the $\mathrm{CaP}$ motor neuron to elicit an action potential (AP) (Fig. 4g, upper trace) and the subsequent EPC was recorded in an innervated muscle fiber in voltage clamp mode (Fig. 4g, lower trace). While quantal content for the AP-evoked EPCs in muscle fibers were not different in mok ${ }^{\mathrm{m} 632-/-}$ larvae (Fig. 4h), we observed variability in EPC amplitude (Fig. $4 \mathrm{i}, \mathrm{j}$ ) and half of the motor neurons tested displayed a higher number of EPC failures. These failures, where CaPs fail to release neurotransmitters, occur at a higher rate during $10 \mathrm{~Hz}, 20 \mathrm{~Hz}$ and 100 $\mathrm{Hz}$ stimulation (traces shown for $1 \mathrm{~Hz}$ in Fig. $4 \mathrm{i}$ and 100 $\mathrm{Hz}$ in Fig. 4j, Fig. 4k, labeled failures in mutant traces, Fig. 4l quantification) (additional frequencies Additional file 9: Figure S9c).

Because we could not perform paired recordings at $2 \mathrm{dpf}$, we then tested if the observed synaptic instability led to locomotion defects at 2 dpf. We performed a touch-evoked escape response (TEER) assay where embryos produce stereotypical swimming episodes in response to touch stimuli (traces of escapes Fig. 5a). The escapes produced by mok m632-/- embryos were of shorter duration and covered less distance than the ones of their wild-type siblings (Fig. 5b). Furthermore, the maximum instant speed, a readout for muscle function [71], was not affected by Dynactin1a depletion (Fig. 5b).

As swimming is a complex behavior that requires synchronous activity of spinal cord neurons, we used optogenetics to exclude the possibility that the observed phenotype arose from impaired circuit connectivity upstream of the CaPs. We probed recruitment of motor neurons in the spinal cord during fictive swimming upon presentation of water jet stimuli (Fig. 5c) while monitoring neuronal activity in the spinal cord with a genetically-encoded calcium indicator (Tg(mnx1:GAL4; UAS:GCaMP5, Fig. 5d). No differences in maximum DF/F amplitude signal for each cell analyzed were noted in 4dpf mok m632-/larvae when compared with their wild type siblings (Fig. 5e). This indicates that CaPs have normal calcium transient when stimulated, and that there are no connectivity defects upstream of the $\mathrm{CaP}$ motor neurons.

These results suggest that Dynactin1a depletion leads to electrophysiological abnormalities at the NMJ, where the synaptic instability observed in $2 \mathrm{dpf}$ CaPs of normal morphology leads to functional deficits at the NMJ culminating in abnormal locomotion, without affecting muscle function, and where $6 \mathrm{dpf}$ mok ${ }^{\mathrm{m} 632-/-}$ larvae displayed varying EPC amplitudes and a higher rate of failures in response to action potential, while still maintaining normal spontaneous release kinetics and quantal content.

\section{Expression of human Dynactin1-eGFP rescues defects in a cell-autonomous manner}

To confirm that the phenotype observed in our mok m632-/- larvae did not involve the muscle fiber or surrounding glia, we specifically overexpressed wild-type human Dynactin1-eGFP (DCTN1-eGFP) together with an E2A-linked membrane-bound fluorophore reporter (tagRFP-Caax) in single $\mathrm{CaP}$ neurons. At 2dpf, overexpression of DCTN1-eGFP did not have an effect on $\mathrm{CaP}$ morphology either in mok m632-/- embryos or their wild-type siblings (Additional file 10: Figure S10 a, b). At 6dpf however, the human protein was able to rescue the morphological phenotype in mok ${ }^{m 632}$ -l- larvae (Fig. 6a), as mutant CaPs had larger, more complex axonal arbors than wild-type CaPs, based on total cell length and number of projections, while maintaining average projection length (Fig. 6b). This overgrowth in rescued CaPs is most likely due to lack of competition from neighboring cells, lacking Dynactin1-eGFP, and still exhibiting a reduced axonal arbor due to Dynactin1a depletion. Overexpression of Dynactin1-eGFP in 6dpf wild-type CaPs did not affect 


\section{a}

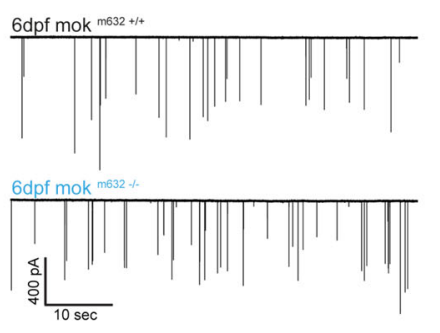

b



d
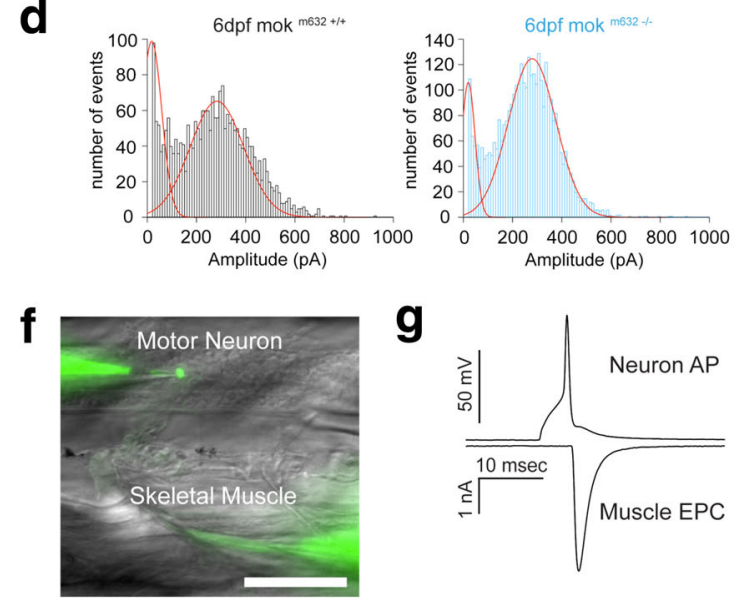

i

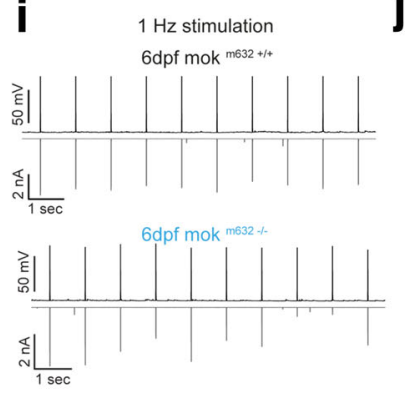

g

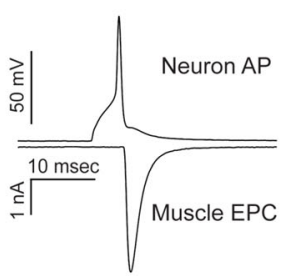

e

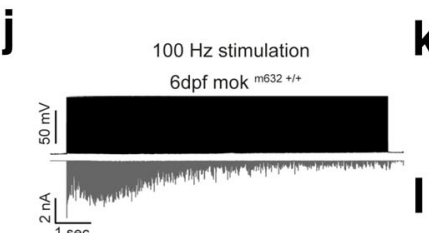

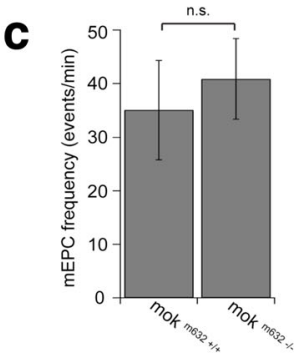

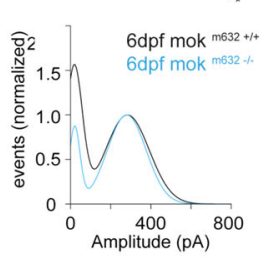

h
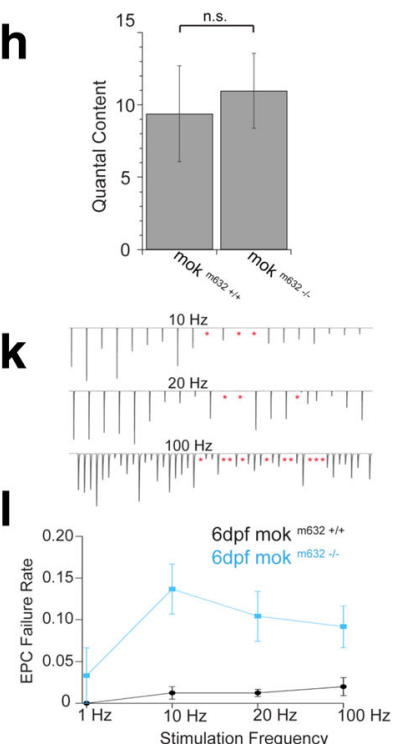

Fig. 4 NMJ function is impaired by severely reduced levels of Dynactin1a. a Sample traces of spontaneous miniature end plate currents (mEPCs) from fast-twitch muscle fibers. $\mathbf{b}$ Normalized individual mEPCs from mok ${ }^{\text {m632-l- }}$ larvae (blue trace) and their wild-type siblings (black trace). $\mathbf{c}$ Average mEPC frequency recorded from wild-type and mutant mok m632-/- larvae. $\mathbf{d}$ mEPC amplitude histogram for wild-type and mok m632-/larvae with Gaussian functions (red traces). e Normalized Gaussian fits from wild-type (black trace) and mok m632-/- larvae (blue trace). $\mathbf{f}$ Paired motor neuron skeletal muscle fiber recording each patched and filled with AlexaFluor 488 (calibration bar = 100um). $\mathbf{g}$ Motor neuron action potential is evoked by a 2 msec current injection (upper trace) and subsequent muscle EPC is recorded (lower trace). $\mathbf{h}$ Average quantal content (evoked EPC amplitude/mEPC amplitude for wild-type and mok ${ }^{\text {m632-l- }}$ larvae. Sample traces from paired neuron - muscle whole cell recordings for wild-type and mok

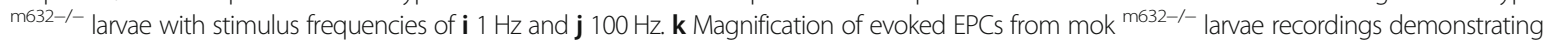
postsynaptic EPC failures occurring at 10, 20 and $100 \mathrm{~Hz}$ (red asterisks). I Evoked EPC failure rate is significantly higher in mok m632-/- (blue trace) than in wild-type larvae (black trace) for paired recordings $10 \mathrm{~Hz}, 20 \mathrm{~Hz}$ and $100 \mathrm{~Hz}$. Data shown as mean $+/-\mathrm{SEM}$. (c: average $=35.04 \pm 9.25, n=2638$ events from 15 fish/ average $=40.86 \pm 7.53, n=4903$ events from 24 fish; $\mathbf{d}: n=2638$ events from 15 fish/ $n=4903$ events from 24 fish; d: WT peak 1 average $=18.35$, WT peak 2 average $=283.19$, mutant peak 1 average $=20.42$, mutant peak 2 average $=280.00 ; \mathbf{h}: Q C=9.39 \pm 3.31, n=8$ pairs $/ \mathrm{QC}=10.98 \pm 2.59, n=18$ pairs; $\mathbf{I}: n=8$ pairs/ $n=9$ pairs)

cell morphology, similar to what was observed at 2 dpf. These results suggest that loss of Dynactin1a is acting in a cell-autonomous manner to cause a morphological phenotype and that human wild-type Dynactin1-eGFP can rescue these defects in mok ${ }^{\mathrm{m} 632}$ ${ }^{-/}$embryos. Furthermore, we observed an enrichment of Dynactin-eGFP at synaptic termini at $6 \mathrm{dpf}$ arguing for a local role at this site (Fig. 6a, c).

\section{Expression of human Dynactin1-GFP rescues behavioral deficits}

To confirm that expression of human wild-type Dynactin1 rescued neuromuscular function in mok ${ }^{\mathrm{m} 632-/-}$ embryos, we synthesized RNA encoding Dynactin1-GFP and injected it in 1-2 cell stage eggs. This resulted in a broad expression of the protein throughout the embryo, as detected by GFP signal (Additional file 10: Figure S10c), 


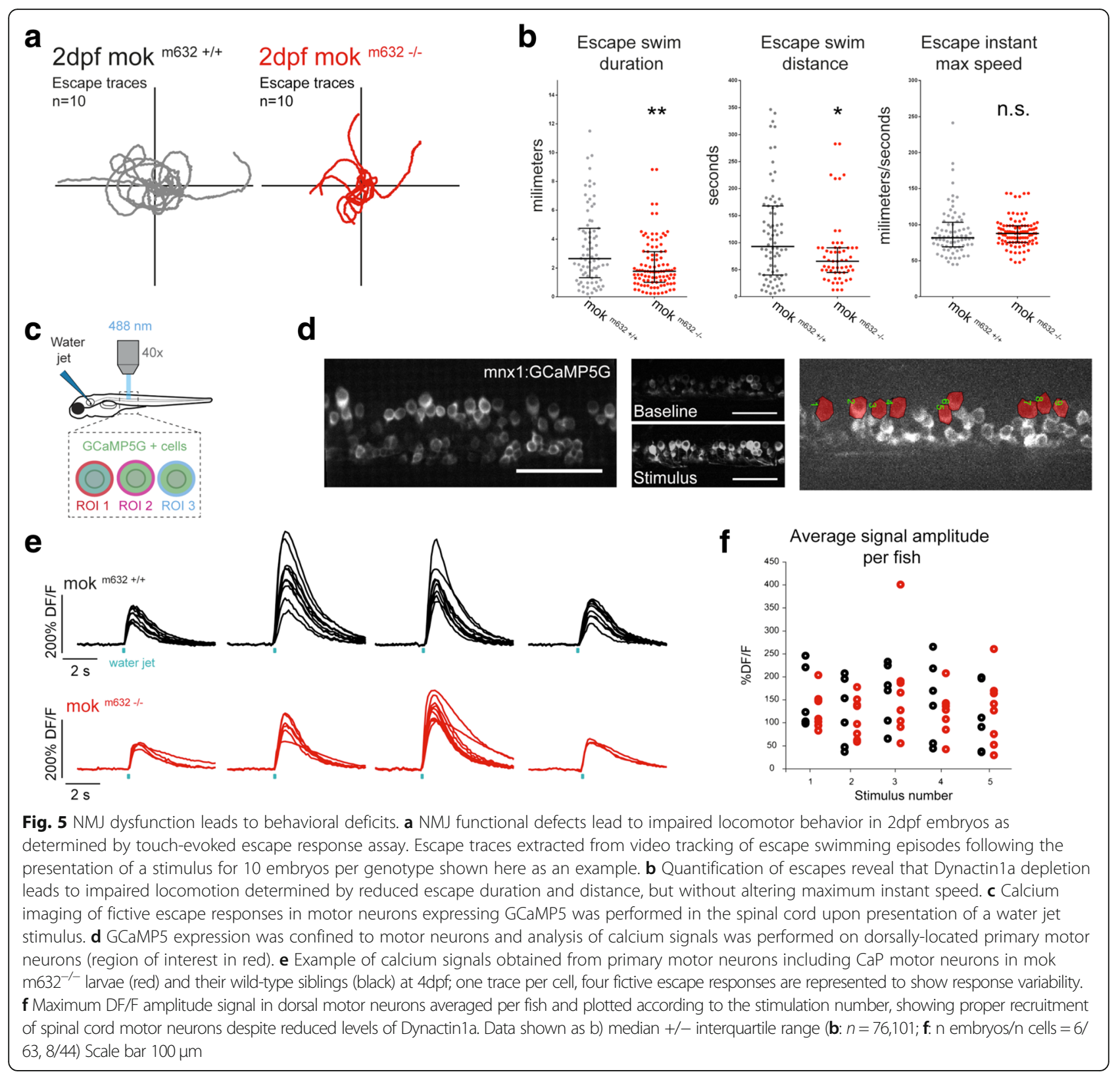

allowing us to perform the 2dpf TEER assay described previously. The escapes produced by mok ${ }^{\mathrm{m} 632-/-}$ and mok ${ }^{\mathrm{m} 632-/+}$ embryos injected with Dynactin1-GFP were of similar duration and distance while being significantly different from uninjected mok ${ }^{\mathrm{m} 632-/-}$ (Fig. 6d). These results show that exogenous Dynactin1-GFP expression can rescue the locomotion abnormalities described in our mutants and thus rescue neuromuscular function.

Overall, our results in CaP motor neurons of the zebrafish spinal cord support a role for Dynactin1a in NMJ dysfunction, where it acts locally to ensure synapse stability and function, independently of its role in regulating dynein activity, directing axonal transport or in modulating cytoskeleton dynamics.

\section{Discussion}

In this study, we characterized a progressive Dynactin1a depletion in vivo, focusing specifically on its effect on primary motor neurons of the zebrafish spinal cord. We report here that loss of Dynactinla is sufficient to impair primary motor neuron function, where $\mathrm{CaP}$ motor neurons exhibit impaired growth of axonal arbors, neuromuscular junction synapse instability and functional abnormalities culminating in locomotion defects.

A striking result of this study is the lack of detectable impact on axonal transport and cytoskeleton dynamics in dynactin1a mutants. It is now well demonstrated that genetic mutations can lead to upregulation of related 


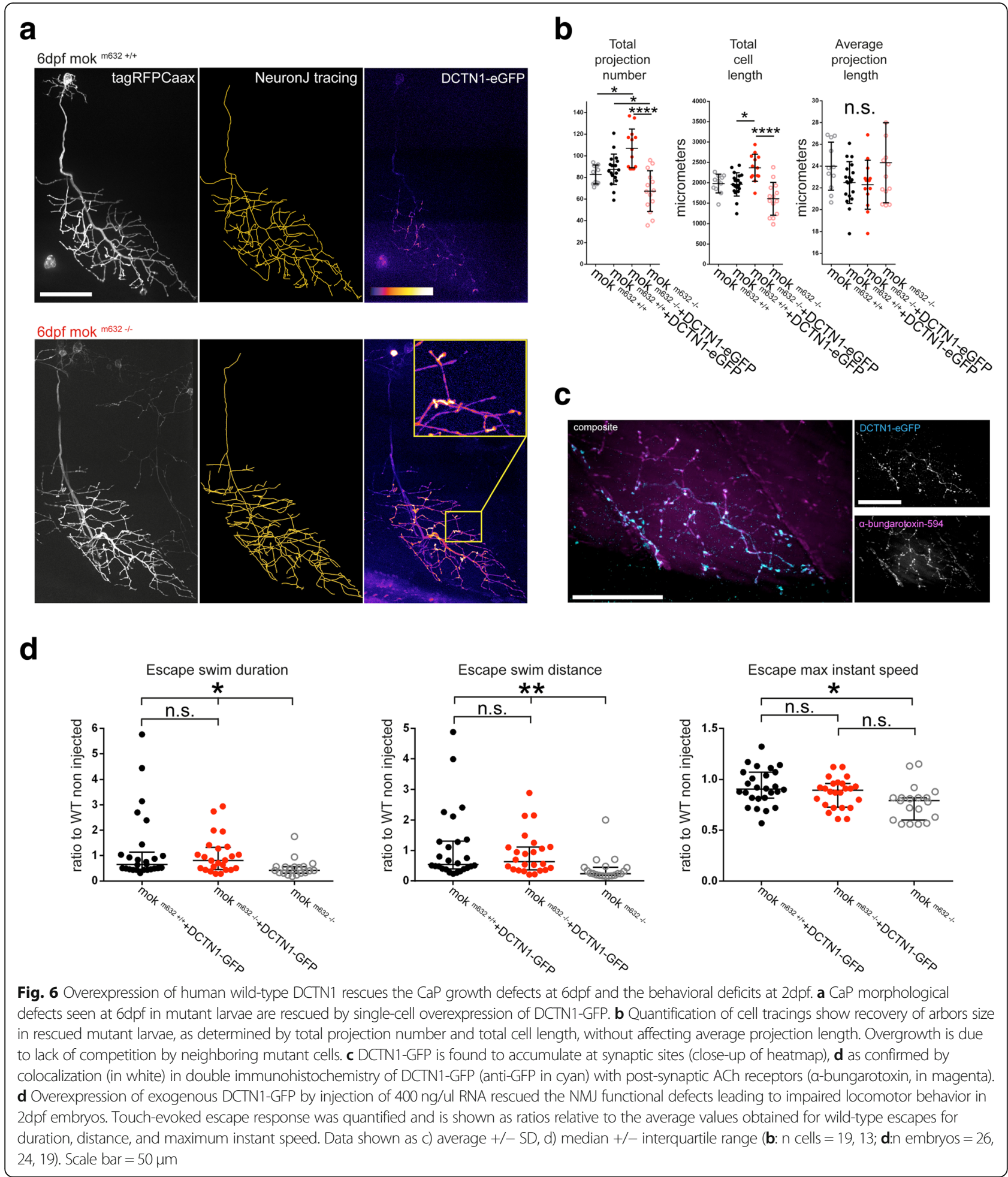

genes and potentially to functional compensation for loss of the protein encoded by the mutated gene [72].

Due to genome duplication, the zebrafish has another para$\log$ for DCTN1: dynactin1b (ZV11; ENSDARG00000056753) that could account for such a compensation. Previous assemblies of the zebrafish genome (ZV8 and ZV9) predict a shorter protein produced from this gene, homologous to the p135 isoform of Dynactin1 [73] lacking the microtubulebinding CAP-Gly domain as a result of alternative splicing. As we did not observe a change in the expression of this paralog 
in $m o k^{m 632-/-}$ larvae by qRT-PCR (Additional file 1: Figure S1d), we conclude that loss of dynactin1a does not trigger a genetic compensation by dynactin $1 b$, however it still could be acting in functional redundancy. We were unable to amplify by RT-PCR a dynactin $1 \mathrm{~b}$ cDNA containing the predicted CAP-GLY domain (exons 1-3) in either $m o k^{m 632-/-}$ or wildtype embryos (data not shown), further supporting the hypothesis that the domain is not included in the wild-type mRNA, and does not get spliced in following dynactin1a silencing. Consequently, dynactin1b likely leads to a protein similar to the $135 \mathrm{kDa}$ isoform, which could explain why we do not see drastic changes in axonal transport. Indeed, both isoforms are found in neuronal populations [74] and bind dynein in independent complexes [73]. Because dynein alone binds well to stable, detyrosinated microtubules, while it requires both the dynactin complex (with the full-sized Dynactin1) and adaptor BicD2 to interact with tyrosinated microtubules such as the ones found at the dynamic (+) ends [75], the absence of full-sized Dynactin1a in presence of the short p135 equivalent Dynactin1b would likely only affect the initiation and not the processivity of transport [76].

We also did not detect changes in modulation of the cytoskeleton dynamics in mok ${ }^{\mathrm{m} 632-/-}$ embryos, another well-described function of Dynactin1 within the dynactin complex. Indeed, our mutants were found to efficiently stabilize microtubules at + ends, necessary for the growth of an axonal arbor, and to capture them at new synapses, a function which rely on interaction of the dynactin complex with the dynein motor and NCAM$180[66,67]$. Following the initial migration of the growth cone to reach its target muscle, the cell relies on cues from the environment in the form of trophic factors acting in a feedback loop with NMJ activity. BDNF is known to act as a retrograde signal to stimulate the maturation of the NMJ synapses by promoting arbor outgrowth and branching and increasing the production of synaptic vesicle proteins [77]. We probed actin filopodia dynamics to determine if the cell was unable to locally detect trophic signaling. These metrics were also found to be unaffected by Dynactin1a depletion, as was the axonal transport of the survival/suicide trophic receptor p75 (ngfra). Furthermore, we did not find altered levels of $b d n f$ mRNA (Additional file 1: Figure S1d), which could have indicated compensation for the growth defects by the muscle target. This suggests that Dynactin1a depletion does not lead to changes in the capacity of the cell to detect or respond to trophic signaling and that our phenotype is independent of modulation of cytoskeleton dynamics.

Although transport and cytoskeleton dynamics appeared unaffected, we detected synapse instability and locomotion abnormalities at $2 \mathrm{dpf}$, before the apparition of morphological defects, suggesting impaired NMJ function. Synapses are known to be necessary for the stabilization of nascent branches of a growing arbor [67]. As CaPs in mok m632-/- larvae have a reduced arbor size, containing a lower number of putative synapses which were of smaller size. Thus, a higher loss of synapses due to instability not compensated by new synapse formation can explain the observed growth defects and the reduction in putative synapse number at $6 \mathrm{dpf}$. While changes in axonal transport or cytoskeleton dynamics would have easily explained the observed phenotype because of the necessity of retrograde transport and cytoskeleton modulation for signaling and degradation, our evidence suggests this is not the mechanism at play upon Dynactinla depletion.

The locomotion defects detected in $2 \mathrm{dpf}$ mok ${ }^{\mathrm{m} 632-/-}$ embryos by behavioral assay (TEER) indicate that the synapse instability observed at this early stage is sufficient to induce functional deficits at the NMJ. Injection of RNA encoding Dynactin1-GFP to obtain a broad but transient overexpression led to the rescue of this behavioral phenotype. It is important to note that the observed locomotion deficits, while statistically significant, were less severe than what has been described in previous ALS models [78-80]. In patients, impairment of NMJ function is reported to arise before the onset of motor neuron degeneration and clinical symptoms in early ALS [81]. This is consistent with our observations as Dynactin1a depletion leads to synapse instability and slight locomotion deficits at $2 \mathrm{dpf}$, before the apparition of a morphological phenotype in $\mathrm{CaP}$ motor neurons and loss of NMJ structural integrity at $6 \mathrm{dpf}$.

Electrophysiological recordings provided a closer look at NMJ synaptic dysfunction, showing that the release machinery is functional at the $2 \mathrm{dpf}$ and $6 \mathrm{dpf}$ mok ${ }^{\mathrm{m} 632}$ ${ }^{-/}$synapses and that the quantity of neurotransmitter contained in individual vesicles was not altered. In addition, no changes were found in the release kinetics or receptor properties. However, paired-recordings of the CaP-fast-twitch muscle fiber at $6 \mathrm{dpf}$ revealed that mok ${ }^{\mathrm{m} 632-/-}$ NMJs have variable amplitudes and a higher rate of response failure to action potentials, when stimulated at 10, 20 and $100 \mathrm{~Hz}$, similar to what has been described for the FUS loss-of-function ALS model [78].

Ultrastructural analysis of the NMJ synapses in $6 \mathrm{dpf}$ mok ${ }^{\text {m632-l }}$ larvae confirmed that we did not have distal accumulation of aggregates, which would be expected in the event of impaired retrograde axonal transport, and showed that the mutant synaptic terminals contained vesicles which were of similar density and average size as the one of their wild-type siblings. We also observed that the active zone perimeter contained similar numbers of available vesicles (readily-releasable), however it was not possible to determine if the formation of synaptic vesicle pools, namely the reserve and recycling pools, was conserved. The synaptic cleft, composed of domains 
connecting the pre- and postsynaptic side of the synapse, was however wider in mok ${ }^{\text {m632-l }}$ larvae at the level of the active zones, suggesting that active zone organization might be disturbed following Dynactin1a depletion. Although we did not investigate this mechanism in more detail, this Dynactin1 could be involved in generating force to maintain membrane apposition by interacting with adhesion molecules or by helping recruit or maintain functional pools in synaptic termini.

In our synapse stability assay, we used Rab3, a protein associated with vesicles at active zones, to label putative synapses. This protein is essential for neurotransmission, with a known role in calcium-evoked exocytosis, and interaction with the SNARE complex [82], and a role in synaptic vesicle formation and trafficking [83]. Moreover, impaired active zone scaffolding could lead to ineffective coupling and recruitment of synaptic vesicles, or could affect calcium channel clustering, explaining the failures in response during repeated action potentials seen in our paired-recordings. Indeed, evoked release (action-potential-mediated) relies on a nanodomain active zone organization for efficient neurotransmission, whereas spontaneous release, which was unaffected in our mutant, can occur at varied areas of the terminal [84]. Hence we speculate that the synapse instability and EPC amplitude variability, as well as the higher failure rate reported here is due to improper formation of active zones following depletion of Dynactin1a, either by interaction with adhesion molecules or by recruitment or maintenance of functional vesicle pools.

Along with the reduced DCNT1 mRNA and protein levels reported in sporadic ALS patients [26], missense mutations in DCTN1 have been reported in ALS patients [28-30,32]. In the context of a dominant inheritance, these missense mutations could likely lead to haploinsufficiency, as one out of three outcomes of missense mutation [85]. In further support of this hypothesis, it was reported that animal models for Dynactin1 mutations lead to a reduction in protein expression, for instance in the G59S mice [50], or the G38S flies [51], however the effect of other ALS-related mutations on protein expression was not studied.

Because many of the ALS-linked mutations were also found in controls $[28,30,32]$, and due to their rarity and the variability of clinical presentation of ALS patients, causality was not established, however it was suggested that these mutations could act as risk factors and compound other rare variants in an oligogenic etiology of ALS [31]. Indeed, DCTN1 expression was found to be downregulated in sporadic ALS patient postmortem spinal cord tissue as an early event preceding degeneration as it was observed in neuronal populations that were well preserved and without pathological markers for degeneration [25]. Our zebrafish model exhibited an initial reduced expression of Dynactin1, followed by a gradual depletion over the course of a few days, thus representing depletion kinetics of interest in the context of investigating the role of this protein in ALS pathogenesis.

\section{Conclusions}

The in vivo characterization of the morphogenesis and function of motor neurons in zebrafish embryos and larvae depleted for Dynactin1a point toward a local role for this protein in stabilizing the neuromuscular synapses, impairing its function, without leading to motor neuron death. This role appears to be independent of Dynactin1's known functions associated with the dynein motor in axonal transport or cytoskeleton dynamics modulation, possibly due to the likely presence of the shorter p135 isoform. Because our probing of interactions with cytoskeletal components or adhesion molecules did not reveal anomalies, a candidate approach of possible synaptic interactors of Dynactin1a would be necessary to help further understand the mechanism at play leading to NMJ dysfunction in this model. The defects reported here are milder than what has been described for established zebrafish ALS models investigating pathogenic mutations, but represent targeted impairments which are consistent with early disease presentation. We therefore propose that Dynactin1a depletion represents an early event in NMJ degeneration and that ALS-related mutations in this gene are likely not causative but indeed have a place in the oligogenic etiology of ALS pathogenesis.

\section{Additional files}

Additional file 1: Figure S1. mok ${ }^{\mathrm{m} 632-/-}$ embryo morphology at $6 \mathrm{dpf}$, Dynactin 1 protein quantification at 2dpf and qRT-PCR expression in mok m632-/- larvae. a) Wild-type sibling and homozygous mutant embryo morphology at $6 \mathrm{dpf}$; close-up showing a dorsal view of the head to emphasize previously described eye phenotype. b) Western blot of maternally-contributed Dynactin1 in 2dpf mok ${ }^{\text {m632-l- }}$ embryo (detected with anti-DCTN1 antibody from Origene, TA346929), c) quantified against gamma-tubulin at 32\% wild-type level. d) Quantification of 3 biological replicates of qRT-PCR levels from $6 \mathrm{dpf}$ mok ${ }^{\mathrm{m} 632-{ }^{-1}}$ larvae mRNA relative to the average wild-type levels obtained for $6 \mathrm{dpf}{ }^{\mathrm{m} 632+/+}$ larvae mRNA (presented as fold change) shows no compensation by dynctin 1b or kif14, no change in the expression of other subunits of the dynactin complex (p22/24, p25, p50, actr1), no change in other known regulators of the dynein motor complex (ndel1b, pafah $1 b 1 a / 1 b 1 b)$, and no changes indicative of trophic compensation (bdnf). (TIF $45716 \mathrm{~kb}$ )

Additional file 2: Figure S2. mok ${ }^{m 632-/-}$ embryo and larvae NMJ structural integrity. a) Double immunohistochemistry reveals the integrity of the NMJ at $2 \mathrm{dpf}$ by coverage and colocalization of presynaptic structures (antisynaptotagmin2, in green) and postsynaptic Ach receptors (a-bungarotoxin, in red). b) Quantification of the colocalization shows normal NMJ structure of the ventral root at $2 \mathrm{dpf}$ by both Pearson's coefficient and the overlap coefficient. c) NMJ structure is also affected at $6 \mathrm{dpf}$, with d) reduced coverage in pre- and postsynaptic components, as well as reduced colocalization. All data presented as average +/- SD; (b: $\mathrm{n}$ embryos = 12, 14; $\mathrm{d}$ : $\mathrm{n}$ larvae = 11,19) (TIF 32575 kb) 
Additional file 3: Figure S3. Dynactin1a depletion does not alter cargo distribution. a) Example of cargo labeling, here for late endosomes (rab7GFP, in green) co-expressed with a membrane-bound reporter (tagRFPCaax, in red) in a single CaP motor neuron. b) The quantification of cargo size and coverage is done by cell compartment (axon, arbor), and distribution from the cell body is analyzed in the axon for mitochondria (phbGFP), early endosomes (rab5c-GFP), late endosomes/multivesicular bodies (rab7-GFP) and recycling endosomes (rab11a-GFP). No difference is found for vesicle or organelle number, mean area, total area or distribution between homozygous mutants and their wild-type siblings at $2 \mathrm{dpf}$. Data shown as average +/- SD. (n cells b: mitochondria $n=7,6$, rab5c $n=5,7$; rab7 $n=7,9$; rab11a $n=10,9$ ). (TIF $19764 \mathrm{~kb}$ )

Additional file 4: Figure S4. Dynactin1a depletion does not alter axonal transport dynamics. a) Example of in vivo timelapse imaging still $(\mathrm{t}=0)$ and extracted kymogram with labelled runs (anterograde cyan, retrograde magenta). b) Kymogram analysis of cargo states reveals no change in dynamics (immobile in black, anterograde in cyan and retrograde in magenta) at $2 \mathrm{dpf}$. In addition, no change was observed in retrograde or anterograde area flux c) and d), or for cargo density e) except for mitochondria which was slightly increased. Data shown as average +/- SEM. ( $n=$ kymogram $c$, d, e: mitochondria $n=5,17$, rab5c $n=7,7$; rab7 $n=11,14$; rab11a $n=7,11$ ). (TIF $24821 \mathrm{~kb}$ )

Additional file 5: Figure S5. Dynactin1a depletion does not alter transport run dynamics. Additional transport metrics for all cargo shows no change in average run speed, length or duration of retrograde and anterograde runs for a) mitochondria, b) rab5c vesicles, c) rab7 vesicles, or d) rab11 vesicles. Data shown as average $+/-$ SEM. $(n=$ kymogram a: $n=5,17 ; \mathrm{b}: n=7,7 ; \mathrm{c}: n=11,14 ; \mathrm{d}: n=7,11)$. (TIF $26989 \mathrm{~kb}$ )

Additional file 6: Figure S6. Transport of p75 receptor (ngfra) is not affected by loss of Dynactin1a. In vivo timelapse imaging of vesicles tagged with ngfra-eGFP fusion protein show no changes in a) transport states, b) area flux and vesicle density, c) average run speed, length and duration of retrograde and anterograde runs of the trophic receptor in $2 \mathrm{dpf}$ CaP motor neurons. All data presented as average +/-SEM. (n kymogram $=10,9)$. (TIF $16813 \mathrm{~kb})$

Additional file 7: Figure S7. Synapse distribution and size at $2 \mathrm{dpf}$ is not affected by loss of Dynactin1a. a) Putative synapses are visualized with rab3-dendra2 labeling in single CaP cells at 2dpf. b) Distribution is not affected, as determined by number, average area and total area, of putative synapses. All data shown as average $+/-\mathrm{SD}(\mathrm{b}$ : $\mathrm{n}$ cells $=26,20)$. (TIF $12772 \mathrm{~kb}$ )

Additional file 8: Figure S8. $\mathrm{N}$-Cadherin localization is not altered at mok $^{\mathrm{m} 632-1-}$ adherens junctions of the NMJ. a) Confocal projection of live 2dpf Tg(cdh2:Cdh2-GFP; mokm632; mnx1:Gal4) embryos showing NCadherin-GFP located th the center of a CaP NMJ synapse, determined by presence of post-synaptic AChR labelled with conjugated abungarotoxin (bath application, in red). b) Whole-mount immunohistochemistry of 6dpf Tg(cdh2:Cdh2-GFP; mokm632; mnx1:Gal4) larvae showing N-Cadherin-GFP (anti-GFP, in green) located at the center of an NMJ synapse, which was co-labeled to show pre-and postsynaptic structures (respectively, synaptotagmin2 in red and conjugated a-bungarotoxin in blue). Boxes show close-up of the synaptic structures, surrounding the $\mathrm{N}$ Cadherin puncta. (TIF $40868 \mathrm{~kb}$ )

Additional file 9: Figure S9. Loss of Dynactin1a leads to abnormal physiological properties of the NMJ. Additional metrics of NMJ whole-cell recordings showing no change in a) Tau decay and b) rise time of 6dpf $\mathrm{mEPC}$ recordings in mok ${ }^{\mathrm{m} 632---}$ larvae. c) Additional frequencies for paired-recordings of CaP-fast twitch muscle fibers at $6 \mathrm{dpf}$ showing similar failure rates of mutant NMJs. (TIF $18204 \mathrm{~kb}$ )

Additional file 10: Figure S10. Overexpression of human wild-type DCTN1 does not affect initial development of CaP motor neurons and exogenous expression is confirmed by increase in GFP signal. a) Overexpression of human wild-type DCTN1-GFP (heatmap), along with a membranebound reporter (tagRFP-Caax, traced in green), is obtained in a cellautonomous manner and does not accumulate at synapses. b) Overexpression does not affect initial growth of CaP motor neurons in either mutant embryos or their wild-type siblings at $2 \mathrm{dpf}$, as determined by total projection number, total cell length and average projection length. c) DCTN1-GFP RNA expression is confirmed by GFP detection in $2 \mathrm{dpf}$ embryos by fluorescent microscopy in the $488 \mathrm{~nm}$ channel, image shown here for non-injected embryos with red bar for histogram generation. Quantification confirms higher fluorescent signal in injected (400 ng/ul DCTN1-GFP) vs non injected wild-type embryos. All data shown as average +/- SD (b: $n=8,14 ; \mathrm{c}: n=10,5)$ (TIF $27508 \mathrm{~kb})$

\section{Abbreviations}

AChR: Acetylcholine receptors; ALS: Amyotrophic lateral sclerosis; AZ: Active zone; CaP: Caudal primary; DCTN1: Dynactin subunit 1; dpf: Days postfertilization; hpf: Hours post-fertilization; mEPCs: Miniature end plate currents; mok $^{\text {m632: }}$ mikre oko ${ }^{\text {m632 }}$; NMJ: Neuromuscular junction; TEER: Touch-evoked escape response

\section{Acknowledgements}

We are grateful to J. Malicki, B. Link, S. Liebau and S. Putz for sharing plasmids and fish lines. We thank the Developmental Biology Curie imaging facility (PICT-IBiSA@BDD, Paris, France, UMR3215/U934) member of the France-Biolmaging national research infrastructure for their help and advice with confocal microscopy. We also thank Asha Baskaran and Dominique Langui from the ICM Quant Imaging Facility for the electron microscopy on larval zebrafish NMJ.

\section{Authors' contributions}

VB, Conception and design, Acquisition of data, Analysis and interpretation of data, Drafting or revising the article. JMH, Acquisition of data, Analysis and interpretation of data. KF, Acquisition of data, Analysis and interpretation of data, revising the article. KD, Acquisition of data, Analysis and interpretation of data. TOA, Contributed unpublished essential data or reagents, revising the article. CR, Contributed unpublished essential data or reagents, revising the article. CW, Conception and design, supervised the project, revising the article. FDB, Conception and design, supervised the project, Drafting or revising the article. All authors read and approved the final manuscript.

\section{Funding}

The Del Bene laboratory "Neural Circuits Development" is part of the Laboratoire d'Excellence (LABEX) entitled DEEP (ANR -11-LABX-0044), and of the École des Neurosciences de Paris lle-de-France network. V. B. was supported by an FRSQ and CIHR Doctoral Award and was enrolled in the ENP Graduate Program. T.O.A. was supported by a Boehringer Ingelheim Fonds Ph.D. fellowship. C.R. was supported by a EU H2020 Marie Skłodowska-Curie Action fellowship (H2020-MSCA-IF-2014 \#661527). This work has been supported by an ATIP/AVENIR program starting grant (F.D.B.), ERC-StG \#311159 (F.D.B.), ERA-NET E-rare grant (for Research Programs on Rare Diseases, 0601165051) (F.D.B.), CNRS, INSERM and Institut Curie core funding, as well as an ERC Starting Grant "Optoloco" \#311673 and the New York Stem Cell Foundation (NYSCF Grant \# NYSCF-R-NI39) for C.W. who is a NYSCF Robertson Investigator.

\section{Availability of data and materials}

The data generated during this study is available upon request.

\section{Ethics approval and consent to participate}

All experiments were performed according to the French and European Union animal welfare guidelines, as well as the Curie Institute ethics protocol.

\section{Consent for publication}

All authors have read the manuscript and indicated consent for publication.

\section{Competing interests}

The authors declare that they have no competing interests.

\section{Author details}

${ }^{1}$ Institut Curie, PSL Research University, INSERM U934, CNRS UMR3215, Sorbonne Université, F-75005 Paris, France. 'Sorbonne Université, Inserm, CNRS, AP-HP, Institut du Cerveau et de la Moelle Épinière, ICM, F-75013 Paris, France. ${ }^{3}$ Present Address: VIB-KU Leuven, Center for Brain \& Disease Research, Leuven, Belgium. ${ }^{4}$ Present Address: Zuckerman Mind Brain Behavior Institute, 
Columbia University, New York, USA. ${ }^{5}$ Present Address: Center for Integrative Genomics, University of Lausanne, Lausanne, Switzerland.

\section{Received: 15 May 2019 Accepted: 10 June 2019 Published online: 10 July 2019}

\section{References}

1. Aren, Reiley, Zarei S, Carr KL, Diaz K, Guerra O, Altamirano PF, et al. A comprehensive review of amyotrophic lateral sclerosis. Surg Neurol Int. 2015;6:171. https://doi.org/10.4103/2152-7806.169561.

2. White MA, Sreedharan J. Amyotrophic lateral sclerosis: recent genetic highlights. Curr Opin Neurol. 2016;29:557-64. https://doi.org/10.1097/WCO. 0000000000000367.

3. Therrien M, Dion PA, Rouleau GA. ALS: recent developments from genetics studies. Curr Neurol Neurosci Rep. 2016;16. https://doi.org/10.1007/s11910016-0658-1.

4. van Es MA, Hardiman O, Chio A, Al-Chalabi A, Pasterkamp RJ, Veldink JH, et al. Amyotrophic lateral sclerosis. Lancet. 2017;390:2084-98. https://doi.org/ 10.1016/S0140-6736(17)31287-4

5. Van Damme P, Robberecht W, Van Den Bosch L. Modelling amyotrophic lateral sclerosis: progress and possibilities. Dis Model Mech. 2017;10:537-49. https://doi.org/10.1242/dmm.029058.

6. Van Blitterswijk M, Van Es MA, Hennekam EAM, Dooijes D, Van Rheenen W Medic J, et al. Evidence for an oligogenic basis of amyotrophic lateral sclerosis. Hum Mol Genet. 2012;21:3776-84. https://doi.org/10.1093/hmg/ dds199.

7. Bettencourt $\mathrm{C}$, Houlden $\mathrm{H}$. News and views exome sequencing uncovers hidden pathways in familial and sporadic ALS. Nat Publ Gr. 2015;18:611-3. https://doi.org/10.1038/nn.4012.

8. Schmeisser MJ, Dupuis L, Roselli F, Shaw PJ, Cooper-Knock J, Robins H, et al. Targeted genetic screen in amyotrophic lateral sclerosis reveals novel genetic variants with synergistic effect on clinical phenotype. Front Mol Neurosci. 2017;10:1-11. https://doi.org/10.3389/fnmol.2017.00370.

9. Chevalier-Larsen E, Holzbaur ELF. Axonal transport and neurodegenerative disease. Biochim Biophys Acta. 2006;1762:1094-108. https://doi.org/10.1016/ j.bbadis.2006.04.002.

10. Duncan JE, Goldstein LSB. The genetics of axonal transport and axonal transport disorders. PLOS Genet. 2006;2:e124. https://doi.org/10.1371/journal. pgen.0020124

11. Soo KY, Farg M, Atkin JD. Molecular motor proteins and amyotrophic lateral sclerosis [Internet]. Int J Mol Sci. 2011:9057-82. https://doi.org/10.3390/ ijms12129057.

12. Perlson E, Maday S, Fu M-M, Moughamian AJ, Holzbaur ELF. Retrograde axonal transport: pathways to cell death? Trends Neurosci. 2010;33:335-44 https://doi.org/10.1016/j.tins.2010.03.006.

13. Millecamps $\mathrm{S}$, Julien J-P. Axonal transport deficits and neurodegenerative diseases. Nat Rev Neurosci. 2013;14:161-76. https:// doi.org/10.1038/nrn3380.

14. Encalada SE, Goldstein LSB. Biophysical challenges to axonal transport: motor-cargo deficiencies and neurodegeneration. Annu Rev Biophys. 2014; 43:141-69. https://doi.org/10.1146/annurev-biophys-051013-022746.

15. Brady ST, Morfini GA. Regulation of motor proteins, axonal transport deficits and adult-onset neurodegenerative diseases. Neurobiol Dis. 2017. https:// doi.org/10.1016/j.nbd.2017.04.010

16. De Vos KJ, Hafezparast M. Neurobiology of axonal transport defects in motor neuron diseases: opportunities for translational research? Neurobiol Dis. 2017. https://doi.org/10.1016/j.nbd.2017.02.004

17. Schnapp BJ, Reese TS. Dynein is the motor for retrograde axonal transport of organelles. Proc Natl Acad Sci U S A. 1989;86:1548-52. https://doi.org/10. 1073/pnas.86.5.1548

18. Kardon J, Vale R. Regulators of the cytoplasmic dynein motor. Nat Rev Mol Cell Biol. 2009:10:854-65. https://doi.org/10.1038/nrm2804.DATABASES

19. Akhmanova A, Hammer JA III. Linking molecular motors to membrane cargo. Curr Opin Cell Biol. 2012;22:479-87. https://doi.org/10.1016/j.ceb.2010 04.008.Linking.

20. Waterman-Storer CM, Karki S, Holzbaur ELF. The p150Glued component of the dynactin complex binds to both microtubules and the actin-related protein centractin (Arp-1). Proc Natl Acad Sci U S A. 1995;92:1634-8 Available: http://www.pubmedcentral.nih.gov/articlerender.fcgi?artid= 42574\&tool=pmcentrez\&rendertype=abstract.
21. Vaughan PS, Miura P, Henderson M, Byrne B, Vaughan KT. A role for regulated binding of $\mathrm{p} 150$ (Glued) to microtubule plus ends in organelle transport. J Cell Biol. 2002;158:305-19. https://doi.org/10.1083/jcb. 200201029

22. Vaughan KT, Vallee RB. Cytoplasmic dynein binds dynactin through a direct interaction between the intermediate chains and p150. J Cell Biol. 1995;131: 1507-16.

23. Chowdhury S, Ketcham SA, Schroer TA, Lander GC. Structural organization of the dynein-dynactin complex bound to microtubules. Nat Struct Mol Biol. 2015:1-6. https://doi.org/10.1038/nsmb.2996.

24. Waterman-Storer CM, Karki SB, Kuznetsov SA, Tabb JS, Weiss DG, Langford $\mathrm{GM}$, et al. The interaction between cytoplasmic dynein and dynactin is required for fast axonal transport. Proc Natl Acad Sci U S A. 1997:94:12180-5 Available: http://www.pubmedcentral.nih.gov/articlerender.fcgi?artid= 23743\&tool=pmcentrez\&rendertype=abstract.

25. Tanaka F, Ikenaka K, Yamamoto M, Sobue G. Neuropathology and omics in motor neuron diseases. Neuropathology. 2012;32:458-62. https://doi.org/10. 1111/j.1440-1789.2011.01281.x.

26. Kuźma-Kozakiewicz M, Chudy A, Kaźmierczak B, Dziewulska D, Usarek E, Barańczyk-Kuźma A. Dynactin deficiency in the CNS of humans with sporadic ALS and mice with genetically determined motor neuron degeneration. Neurochem Res. 2013. https://doi.org/10.1007/s11064-0131160-7.

27. Puls I, Jonnakuty C, LaMonte B, Holzbaur ELF, Tokito MK, Mann E, et al. Mutant dynactin in motor neuron disease. Nat Genet. 2003;33:455-6. https://doi.org/10.1038/ng1123.

28. Münch C, Sedlmeier R, Meyer T, Homberg V, Sperfeld AD, Kurt A, et al. Point mutations of the p150 subunit of dynactin (DCTN1) gene in ALS. Neurology, Available. 2004;63:724-6 http://www.ncbi.nlm.nih.gov/pubmed/15326253.

29. Münch C, Rosenbohm A, Sperfeld AD, Uttner I, Reske S, Krause BJ, et al. Heterozygous R1101K mutation of the DCTN1 gene in a family with ALS and FTD. Ann Neurol. 2005:58:777-80. https://doi.org/10.1002/ana.20631.

30. Stockmann M, Meyer-Ohlendorf M, Achberger K, Putz S, Demestre M, Yin H, et al. The dynactin p150 subunit: cell biology studies of sequence changes found in ALS/MND and parkinsonian syndromes. J Neural Transm. 2013;120: 785-98. https://doi.org/10.1007/s00702-012-0910-z.

31. Liu X, Yang L, Tang L, Chen L, Liu X, Fan D. DCTN1 gene analysis in Chinese patients with sporadic amyotrophic lateral sclerosis. PLoS One. 2017;12:1-8. https://doi.org/10.1371/journal.pone.0182572.

32. Vilariño-Güell C, Wider C, Soto-Ortolaza Al, Cobb SA, Kachergus JM, Keeling $\mathrm{BH}$, et al. Characterization of DCTN1 genetic variability in neurodegeneration. Neurology. 2009;72:2024-8. https://doi.org/10.1212/ WNL.0b013e3181a92c4c

33. Moughamian AJ, Holzbaur ELF. Dynactin Is Required for Transport Initiation from the Distal Axon. Neuron. 2012;74:331-43. https://doi.org/10.1016/j. neuron.2012.02.025

34. Levy JR, Sumner CJ, Caviston JP, Tokito MK, Ranganathan S, Ligon LA, et al. A motor neuron disease-associated mutation in p150Glued perturbs dynactin function and induces protein aggregation. J Cell Biol. 2006;172: 733-45. https://doi.org/10.1083/jcb.200511068.

35. Finsterer J, Burgunder J-M. Recent progress in the genetics of motor neuron disease. Eur J Med Genet. 2014;57:103-12. https://doi.org/10.1016/j.ejmg 2014.01.002.

36. Zelenchuk TA, Brusés JL. In vivo labeling of zebrafish motor neurons using an mnx1 enhancer and Gal4/UAS. Genesis. 2011:49:546-54. https://doi.org/ 10.1002/dvg.20766.

37. Malicki J, Neuhauss SCF, Schier AF, Solnica-krezel L, Stemple DL, Stainier DYR, et al. Mutations affecting development of the zebrafish retina. Development. 1996;123:263-73 Available: http://dev.biologists.org/content/ 123/1/263.short.

38. Doerre G, Malicki J. A mutation of early photoreceptor development, mikre oko, reveals cell-cell interactions involved in the survival and differentiation of zebrafish photoreceptors. J Neurosci. 2001;21:6745-57 Available: http:// www.ncbi.nlm.nih.gov/pubmed/11517263.

39. Tsujikawa M, Omori Y, Biyanwila J, Malicki J. Mechanism of positioning the cell nucleus in vertebrate photoreceptors. Proc Natl Acad Sci U S A. 2007; 104:14819-24. https://doi.org/10.1073/pnas.0700178104.

40. Akerboom J, Chen T-W, Wardill TJ, Tian L, Marvin JS, Mutlu S, et al. Optimization of a GCaMP calcium Indicator for neural activity imaging. J Neurosci. 2012:32:13819-40. https://doi.org/10.1523/JNEUROSCI.2601-12. 2012. 
41. Revenu C, Streichan S, Dona E, Lecaudey V, Hufnagel L, Gilmour D. Quantitative cell polarity imaging defines leader-to-follower transitions during collective migration and the key role of microtubule-dependent adherens junction formation. Development. 2014;141:1282-91. https://doi. org/10.1242/dev.101675.

42. Clark B, Winter M, Cohen A, Link B. Generation of Rab-based transgenic lines for in vivo studies of endosome biology in zebrafish. Dev Dyn. 2011;240: 2452-65. https://doi.org/10.1002/dvdy.22758.Generation.

43. Kwan KM, Fujimoto E, Grabher C, Mangum BD, Hardy ME, Campbell DS, et al. The Tol2kit: a multisite gateway-based construction Kit for Tol2 transposon transgenesis constructs. Dev Dyn. 2007;236:3088-99. https://doi. org/10.1002/dvdy.21343

44. Auer TO, Xiao T, Bercier V, Gebhardt C, Duroure K, Concordet J-P, et al. Deletion of a kinesin I motor unmasks a mechanism of homeostatic branching control by neurotrophin-3. Elife. 2015;4:1-26. https://doi.org/10. 7554/eLife.05061.

45. Campbell DS, Stringham SA, Timm A, Xiao T, Law MY, Baier H, et al. Slit1a inhibits retinal ganglion cell Arborization and synaptogenesis via Robo2dependent and -independent pathways. Neuron. 2007:55:231-45. https:// doi.org/10.1016/j.neuron.2007.06.034.

46. Preibisch S, Saalfeld S, Tomancak P. Globally optimal stitching of tiled $3 D$ microscopic image acquisitions. Bioinformatics. 2009;25:1463-5. https://doi. org/10.1093/bioinformatics/btp184.

47. Tang R, Dodd A, Lai D, McNabb WC, Love DR. Validation of zebrafish (Danio rerio) reference genes for quantitative real-time RT-PCR normalization. Acta Biochim Biophys Sin Shanghai. 2007;39:384-90. https://doi.org/10.1111/j. 1745-7270.2007.00283.x.

48. Livak KJ, Schmittgen TD. Analysis of relative gene expression data using real-time quantitative PCR and the 2(-Delta Delta C(T)) method. Methods. 2001;25:402-8. https://doi.org/10.1006/meth.2001.1262.

49. Lattante S, Rouleau GA, Kabashi E. TARDBP and FUS Mutations Associated with Amyotrophic Lateral Sclerosis : Summary and update. Hum Mutat. 2013;34:812-26. https://doi.org/10.1002/humu.

50. Lai C, Lin X, Chandran J, Shim H, Yang W-J, Cai H. The G59S mutation in p150glued causes dysfunction of dynactin in mice. J Neurosci. 2007;27: 13982-90. https://doi.org/10.1523/JNEUROSCl.4226-07.2007.

51. Lloyd TE, Machamer J, O'Hara K, Kim JH, Collins SE, Wong MY, et al. The p150(Glued) CAP-Gly domain regulates initiation of retrograde transport at synaptic termini. Neuron. 2012;74:344-60. https://doi.org/10.1016/j.neuron. 2012.02.026.

52. Del Bene F, Wehman AMA, Link BBA, Baier H, Del Bene F, Wehman AMA, et al. Regulation of neurogenesis by interkinetic nuclear migration through an apical-basal Notch gradient. Cell. 2007;134:1055-65. https://doi.org/10.1016/j. cell.2008.07.017.Regulation.

53. Moughamian AJ, Osborn GE, Lazarus JE, Maday S, Holzbaur ELF. Ordered recruitment of dynactin to the microtubule plus-end is required for efficient initiation of retrograde axonal transport. J Neurosci. 2013;33:13190-203. https://doi.org/10.1523/JNEUROSCI.0935-13.2013.

54. Myers PZ, Eisen JS, Westerfield M. Development and axonal outgrowth of identified motoneurons in the zebrafish. J Neurosci Soc Neuroscience. 1986; 6:2278-89 Available: http://www.ncbi.nlm.nih.gov/pubmed/3746410.

55. Jackson HE, Ingham PW. Control of muscle fibre-type diversity during embryonic development: the zebrafish paradigm. Mech Dev. 2013;130:44757. https://doi.org/10.1016/j.mod.2013.06.001.

56. Flanagan-Steet H, Fox MA, Meyer D, Sanes JR. Neuromuscular synapses can form in vivo by incorporation of initially aneural postsynaptic specializations. Development. 2005;132:4471-81. https://doi.org/10.1242/dev.02044.

57. Marjan H, Cavalli V, Shah SB, Schimmelpfeng K, Brusch R, Yang G, et al. Dynactin is required for coordinated bidirectional motility, but not for dynein membrane attachment. Mol Biol Cell. 2007;18:2081-9.

58. Jing L, Lefebvre $J$, Gordon LR, Granato M. Wht signals organize synaptic prepattern and axon guidance through the zebrafish unplugged/MuSK receptor. Neuron. 2009;61:721-33. https://doi.org/10. 1016/j.neuron.2008.12.025

59. Bonanomi D, Pfaff SL. Motor axon pathfinding. Cold Spring Harb Perspect Biol. 2010;2:1-19. https://doi.org/10.1101/cshperspect.a001735.

60. Smith RB, Machamer JB, Kim NC, Hays TS, Marques G. Relay of retrograde synaptogenic signals through axonal transport of BMP receptors. J Cell Sci. 2012;125:3752-64. https://doi.org/10.1242/jcs.094292.

61. Cosker KE, Pazyra-Murphy MF, Fenstermacher SJ, Segal RA. Target-derived neurotrophins coordinate transcription and transport of Bclw to prevent axonal degeneration. Ann Intern Med. 2013;158:5195-207. https://doi.org/ 10.1523/JNEUROSCI.3862-12.2013.

62. Sanes JR, Lichtman JW. Development of the vertebrate neuromuscular junction. Annu Rev Neurosci. 1999;22:389-442.

63. Baas PW, Nadar CV, Myers KA. Axonal transport of microtubules: the long and short of it. Traffic. 2006;7:490-8. https://doi.org/10.1111/j.1600-0854. 2006.00392.x.

64. Panzer JA, Song Y, Balice-Gordon RJ. In vivo imaging of preferential motor axon outgrowth to and synaptogenesis at prepatterned acetylcholine receptor clusters in embryonic zebrafish skeletal muscle. J Neurosci. 2006;26:934-47. https://doi.org/10.1523/JNEUROSCI.3656-05. 2006.

65. Bearce EA, Erdogan B, Lowery LA. TIPsy tour guides: how microtubule plusend tracking proteins (+TIPs) facilitate axon guidance. Front Cell Neurosci. 2015:9:1-12. https://doi.org/10.3389/fncel.2015.00241.

66. Lazarus JE, Moughamian AJ, Tokito MK, Holzbaur ELF. Dynactin subunit p150(glued) is a neuron-specific anti-catastrophe factor. PLoS Biol. 2013;11: e1001611. https://doi.org/10.1371/journal.pbio.1001611.

67. Meyer MP, Smith SJ. Evidence from in vivo imaging that synaptogenesis guides the growth and branching of axonal arbors by two distinct mechanisms. J Neurosci. 2006:26:3604-14. https://doi.org/10.1523/ JNEUROSCI.0223-06.2006

68. Chang L, Kreko T, Davison H, Cusmano T, Wu Y, Rothenfluh A, et al. Normal dynactin complex function during synapse growth in Drosophila requires membrane binding by Arfaptin. Mol Biol Cell. 2013;24:1749-64, S1-5. https:// doi.org/10.1091/mbc.E12-09-0697.

69. Eaton BA. Fetter RD, Davis GW. Dynactin is necessary for synapse stabilization. Neuron. 2002;34:729-41 Available: http://www.ncbi.nlm.nih. gov/pubmed/12062020.

70. Wen $\mathrm{H}$, Brehm P. Paired motor neuron-muscle recordings in zebrafish test the receptor blockade model for shaping synaptic current. J Neurosci. 2005;25:8104-11. https://doi.org/10.1523/JNEUROSCI.2611-05. 2005

71. Sztal TE, Ruparelia AA, Williams C, Bryson-Richardson RJ. Using touch-evoked response and locomotion assays to assess muscle performance and function in zebrafish. J Vis Exp. 2016:1-6. https://doi.org/10.3791/54431.

72. El-Brolosy MA, Kontarakis Z, Rossi A, Kuenne C, Günther S, Fukuda N, et al. Genetic compensation triggered by mutant mRNA degradation. Nature. 2019;568:193-7. https://doi.org/10.1038/s41586-019-1064-z.

73. Tokito MK, Howland DS, Lee VM, Holzbaur ELF. Functionally distinct isoforms of dynactin are expressed in human neurons. Mol Biol Cell. 1996;7: 1167-80 Available: http://www.pubmedcentral.nih.gov/articlerender. fcgi?artid=275970\&tool=pmcentrez\&rendertype=abstract.

74. Carter AP, Diamant AG, Urnavicius L. How dynein and dynactin transport cargos: a structural perspective. Curr Opin Struct Biol. 2016;37:62-70. https:// doi.org/10.1016/j.sbi.2015.12.003.

75. Dubey J, Ratnakaran N, Koushika SP. Neurodegeneration and microtubule dynamics: death by a thousand cuts. Front Cell Neurosci. 2015:1-15. https:// doi.org/10.3389/fncel.2015.00343.

76. McKenney RJ, Huynh W, Vale RD, Sirajuddin M. Tyrosination of a-tubulin controls the initiation of processive dynein-dynactin motility. EMBO J. 2016: 35:e201593071. https://doi.org/10.15252/embj.201593071.

77. Lu B, Je HS. Neurotrophic regulation of the development and function of the neuromuscular synapses. J Neurocytol. 2003;32:931-41. https://doi.org/ 10.1023/B:NEUR.0000020633.93430.db.

78. Armstrong GAB, Drapeau P. Loss and gain of FUS function impair neuromuscular synaptic transmission in a genetic model of ALS. Hum Mol Genet. 2013; Available: http://hmg.oxfordjournals.org/content/early/2013/06/ 13/hmg.ddt278.short.

79. Kabashi E, Lin L, Tradewell ML, Dion PA, Bercier V, Bourgouin P, et al. Gain and loss of function of ALS-related mutations of TARDBP (TDP-43) cause motor deficits in vivo. Hum Mol Genet. 2009;19:671-83. https://doi.org/10. 1093/hmg/ddp534.

80. Kabashi E, Bercier V, Lissouba A, Liao M, Brustein E, Rouleau GA, et al. Fus and tardbp but not sod 1 interact in genetic models of amyotrophic lateral sclerosis. PLoS Genet. 2011;7:17-28. https://doi.org/10.1371/journal.pgen. 1002214.

81. Moloney EB, de Winter F, Verhaagen J. ALS as a distal axonopathy: molecular mechanisms affecting neuromuscular junction stability in the presymptomatic stages of the disease. Front Neurosci. 2014;8:252. https:// doi.org/10.3389/fnins.2014.00252. 
82. Rizo J, Xu J. The synaptic vesicle release machinery. Annu Rev Biophys. 2015;44:339-67. https://doi.org/10.1146/annurev-biophys-060414-034057.

83. Tanaka M, Miyoshi J, Ishizaki H, Togawa A, Ohnishi K, Endo K, et al. Role of Rab3 GDP/GTP exchange protein in synaptic vesicle trafficking at the mouse neuromuscular junction. Mol Biol Cell. 2001;12:1421-30. https://doi.org/10. 1091/mbc.12.5.1421

84. Biederer T, Kaeser PS, Blanpied TA. Transcellular Nanoalignment of Synaptic Function. Neuron. 2017;96:680-96. https://doi.org/10.1016/..neuron.2017.10.006.

85. Hijikata A, Tsuji T, Shionyu M, Shirai T. Decoding disease-causing mechanisms of missense mutations from supramolecular structures. Sci Rep. 2017;7:1-8. https://doi.org/10.1038/s41598-017-08902-1.

\section{Publisher's Note}

Springer Nature remains neutral with regard to jurisdictional claims in published maps and institutional affiliations.

Ready to submit your research? Choose BMC and benefit from:

- fast, convenient online submission

- thorough peer review by experienced researchers in your field

- rapid publication on acceptance

- support for research data, including large and complex data types

- gold Open Access which fosters wider collaboration and increased citations

- maximum visibility for your research: over $100 \mathrm{M}$ website views per year

At BMC, research is always in progress.

Learn more biomedcentral.com/submissions 